\title{
Angiopoietin-I-expressing adipose stem cells genetically modified with baculovirus nanocomplex: investigation in rat heart with acute infarction
}

\author{
This article was published in the following Dove Press journal: \\ International Journal of Nanomedicine \\ 7 February 2012 \\ Number of times this article has been viewed
}

\author{
Arghya Paul' \\ Madhur Nayan² \\ Afshan Afsar Khan' \\ Dominique Shum-Tim² \\ Satya Prakash' \\ 'Biomedical Technology and Cell \\ Therapy Research Laboratory, \\ Department of Biomedical \\ Engineering, Faculty of Medicine, \\ McGill University, ${ }^{2}$ Divisions of \\ Cardiac Surgery and Surgical \\ Research, The Montreal General \\ Hospital, Montreal, QC, Canada
}

Correspondence: Satya Prakash Biomedical Technology and Cell Therapy Research Laboratory, Department of Biomedical Engineering, Faculty of Medicine, 3775 University Street, McGill University, Montreal, QC H3A 2B4, Canada

$\mathrm{Tel}+\mathrm{I} 5$ I4 3983676

Fax + I 5 |4 398746 I

Email satya.prakash@mcgill.ca

\begin{abstract}
The objective of this study was to develop angiopoietin-1 (Ang1)-expressing genetically modified human adipose tissue derived stem cells (hASCs) for myocardial therapy. For this, an efficient gene delivery system using recombinant baculovirus complexed with cell penetrating transactivating transcriptional activator TAT peptide/deoxyribonucleic acid nanoparticles (Bac-NP), through ionic interactions, was used. It was hypothesized that the hybrid Bac-NP $\mathrm{Ang1}_{1}$ system can efficiently transduce hASCs and induces favorable therapeutic effects when transplanted in vivo. To evaluate this hypothesis, a rat model with acute myocardial infarction and intramyocardially transplanted Ang1-expressing hASCs (hASC-Ang1), genetically modified by Bac-NP $\mathrm{Ang}_{1}$, was used. Ang1 is a crucial pro-angiogenic factor for vascular maturation and neovasculogenesis. The released hAng1 from hASC-Ang1 demonstrated profound mitotic and anti-apoptotic activities on endothelial cells and cardiomyocytes. The transplanted hASCAng1 group showed higher cell retention compared to hASC and control groups. A significant increase in capillary density and reduction in infarct sizes were noted in the infarcted hearts with hASC-Ang1 treatment compared to infarcted hearts treated with hASC or the untreated group. Furthermore, the hASC-Ang1 group showed significantly higher cardiac performance in echocardiography (ejection fraction $46.28 \% \pm 6.3 \%, P<0.001$ versus control, $\mathrm{n}=8$ ) than the hASC group $(36.35 \% \pm 5.7 \%, P<0.01, \mathrm{n}=8)$, 28 days post-infarction. The study identified Bac-NP complex as an advanced gene delivery vehicle for stem cells and demonstrated its potential to treat ischemic heart disease with high therapeutic index for combined stem cell-gene therapy strategy.
\end{abstract}

Keywords: combined stem cell-gene therapy, baculovirus, nanoparticle, myocardial therapy, angiogenesis, tissue engineering

\section{Introduction}

A promising therapeutic approach currently under intensive clinical trials is mesenchymal stem cell (MSC) therapy for congestive heart diseases, which depends on several corroborated mechanisms, such as myocyte formation and neovascularization to improve cardiac function and attenuate ventricular remodeling. ${ }^{1,2}$ Despite promising initial results, such clinical application remains limited due to logistic, economic, and timing issues when harvesting autologous stem cells from elderly patients. Moreover, it has been reported that MSCs obtained from elderly donors and patients with diabetes or ischemic heart disease have a significantly reduced capacity for neovascularization, proliferation, and differentiation potential. ${ }^{3}$ 
Delivering proangiogenic proteins, such as vascular endothelial growth factor (VEGF) and angiopoietin-1 (Ang1), using MSC-based gene therapy approaches are currently being employed in recent studies as an alternative strategy to promote myocardial angiogenesis and regeneration..$^{4-6}$ However, the mammalian gene delivery vectors widely used in such studies have various drawbacks and severe safety concerns..$^{7-9}$ These vectors are prone to integration into coding regions of transcriptionally active genes, raising concerns about gene silencing and insertional mutagenesis. On the other hand, nonviral gene delivery systems are much safer for potential clinical applications, but are currently limited by low transfection efficiency and highly transient nature of expression with suboptimal level of transgene expression. Unlike the aforementioned vectors, baculovirus (Bac) is an insect cell-originated viral vector that is considered nonpathogenic to humans as they cannot replicate in mammalian cells. ${ }^{9}{ }^{10}$ The viral genome remains in episomal form within the nucleus and is degraded within mammalian cells over time, eliminating the chance of potential mutagenesis. They can efficiently transduce a wide range of mammalian cells with minimal cytotoxicity, including MSCs as shown in earlier studies, and reports confirm the safety of transplanting Bac-engineered MSCs into immunocompetent animals for cell-based gene therapy. ${ }^{9,11}$

The present study aimed to develop a hybrid nanodelivery system to genetically modify MSCs efficiently, utilizing the complementary strengths of Bac, such as relatively high transduction efficiency and easy scale-up, and transactivating transcriptional activator (TAT)/deoxyribonucleic acid (DNA) nanoparticles (NPs), such as low immunogenicity. TAT peptide sequence, obtained from the protein transduction domain of human immunodeficiency virus-1 responsible for nuclear import of human immunodeficiency virus genome, was modified here by incorporating histidine and cysteine residues for enhanced DNA transport, efficient cell penetration, cell vesicle escape, and transgene expression. ${ }^{12}$ The potential of this new system for direct gene therapy with cardiomyocytes has recently been demonstrated. ${ }^{13}$ Here, it was hypothesized that MSC transduction efficiency can also be significantly enhanced by this new system where the negatively charged Bac is coupled with positively charged endosomolytic histidine rich TAT peptide/DNA NPs, both carrying the transgene. For this, a unique pool of MSCs located within the adipose tissue was used, called adipose tissue-derived stem cells (ASCs), mainly because of their practical availability and pro-angiogenic, immunomodulatory, and other unique properties. ${ }^{14,15}$ Based on the positive efficacy data in disease-relevant animal models, ASCs have recently entered into their first clinical trial. ${ }^{16}$ In a recent intriguing study, Metzele et al reported that human ASCs (hASCs) can fuse to newborn rat heart cells to form new cardiomyocytes with several nuclei, which can beat when maintained in a culture environment. ${ }^{17} \mathrm{ASC}$ s possess a natural ability to secrete VEGF, which overexpresses under hypoxic conditions in ischemic tissues. ${ }^{18}$

It has been reported that VEGF in cooperation with another angiogenic growth factor, Ang1, promotes significant neovascularization, and their combined action leads to a formation of mature and functional vasculature. ${ }^{19-21}$ Thus, it was hypothesized that the secretion of Ang 1 along with naturally releasing VEGF from the genetically modified ASCs, together with their inherent transdifferentiation abilities to cardiomyocytes, can induce a superior synergistic therapeutic effect for myocardial regeneration therapy. Additionally, acute myocardial infarction also induces a high circulating endogenous serum VEGF state. ${ }^{22}$ With these rationales, Ang1 complementary DNA-carrying recombinant Bac and TAT NPs were generated, a self-assembled binary nanocomplex was prepared by hybridizing the two, and its efficiency to express functionally active Ang1 was determined using optimized transduction protocol with hASCs. The in vivo efficacy of the formulated nanobiohybrid (Bac-NP) system for combined stem cell-gene therapy applications was also evaluated using human Ang1 (hAng1)-expressing genetically modified hASCs for myocardial therapy using an immunocompetent infarcted rat heart model. A schematic presentation of the entire procedure is demonstrated in Figure 1.

\section{Methods and materials Cell culture and preparation of Bac gene delivery complex}

Human ASCs ( $\mathrm{n}=1$ male donor) were obtained from Invitrogen Life Technologies (Carlsbad, CA) and cultured in Gibco ${ }^{\circledR}$ Dulbecco's Modified Eagle Medium (Invitrogen) supplemented with $10 \%$ fetal bovine serum (FBS, Gibco, Grand Island, NY). The cells were routinely maintained as stationary cultures in $75 \mathrm{~cm}^{2}$ tissue culture flasks (Corning, MA) and incubated at $37^{\circ} \mathrm{C}$ in a controlled environment with an air atmosphere of $5 \%$ carbon dioxide. Human umbilical vein endothelial cells (HUVECs) (ScienCell Research Laboratories, Carlsbad, CA) were cultured and expanded on tissue culture flasks according to the supplier's instructions. They were cultured in endothelial cell medium (ScienCell) supplemented with 5\% FBS and placed in an incubator containing $5 \%$ carbon dioxide at $37^{\circ} \mathrm{C}$. $\mathrm{H} 9 \mathrm{c} 2$ myogenic cell line, derived from embryonic rat heart 


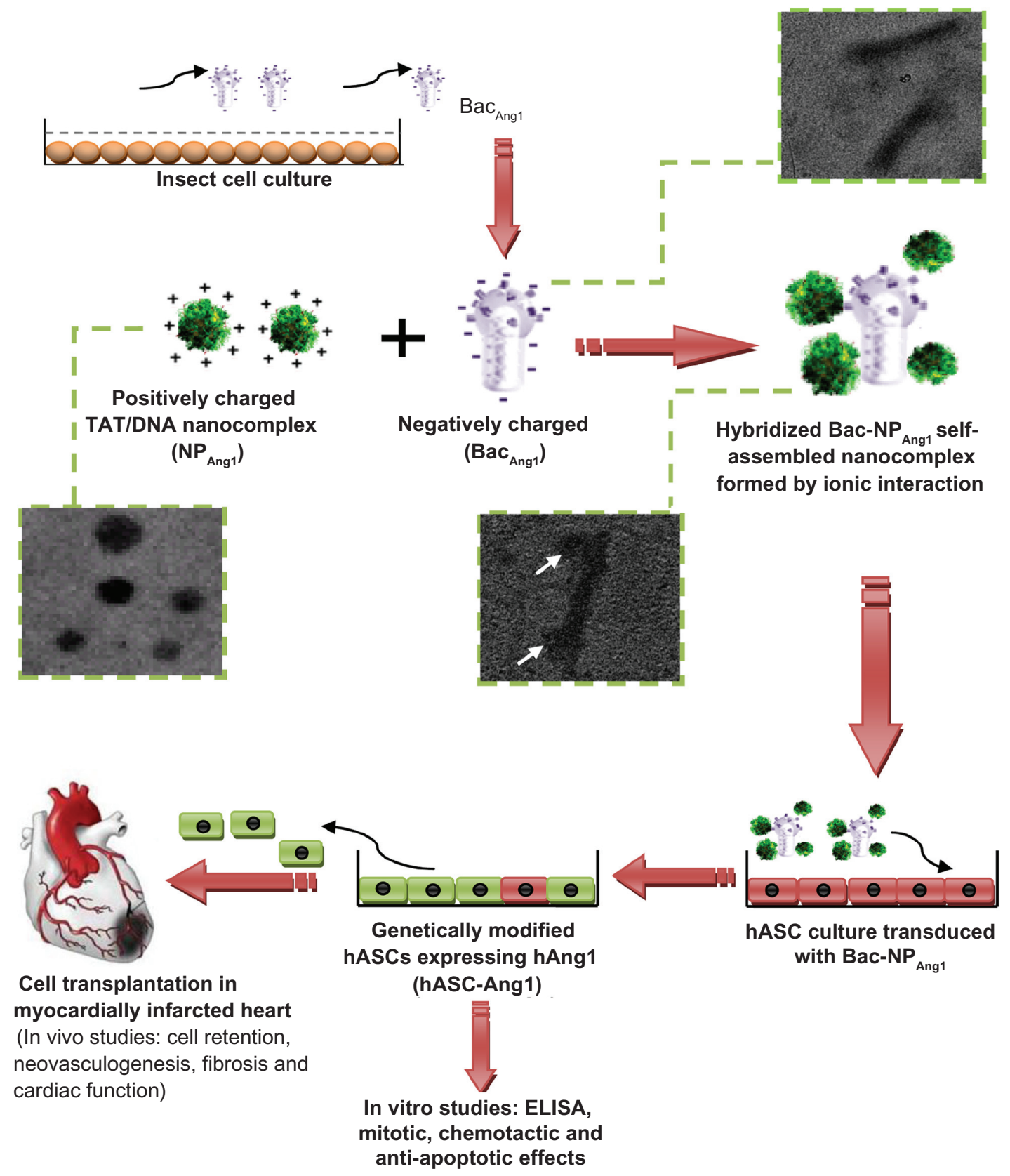

Figure I Schematic representation of the overall scheme: generation of recombinant baculovirus (Bac ${ }_{\text {Ang }}$ ), preparation of hybridized baculovirus with TAT/DNA nanoparticles, in vitro human adipose tissue-derived stem cell transduction, and in vivo investigation using direct intramyocardial transplantation of ASC-Ang I using a rat model with acute myocardial infarction. Transmission electronic microscopic pictures of baculovirus, nanoparticle, and baculovirus-nanoparticle complex shown in subsets confirm the formation of the nanocomplex (in white arrows).

Abbreviations: ASC-AngI, angiopoietin-I-expressing adipose tissue-derived stem cells; $\mathrm{Bac}_{\mathrm{Ang}}$, angiopoietin-I-carrying baculovirus; Bac-NP ${ }_{\text {Angl }}$, angiopoietin-Icarrying baculovirus-nanoparticle complex; DNA, deoxyribonucleic acid; ELISA, enzyme-linked immunosorbent assay; $\mathrm{NP}_{\text {Angl, }}$ angiopoietin-I-carrying nanoparticles; TAT, transactivating transcriptional activator.

ventricles, was obtained from American Type Culture Collection (CRL-1446; Manassas, VA) and cultured in Dulbecco's Modified Eagle Medium supplemented with 10\% FBS. The cells were routinely maintained as stationary cultures in flasks, incubated at $37^{\circ} \mathrm{C}$ in a controlled environment with $5 \%$ carbon dioxide. Sf 9 insect cells (Invitrogen) were maintained at $27^{\circ} \mathrm{C}$ in SF-900 ${ }^{\mathrm{TM}}$ III serum free medium (Invitrogen). The cells were maintained in exponential growth phase and subcultured twice per week. Larger volumes were prepared in shaker flasks (Erlenmeyer; Corning Life Sciences, Lowell, MA) which were agitated at $120 \mathrm{rpm}$ in an incubator shaker as mentioned in earlier studies. ${ }^{11}$

Generation of LacZ and Angl gene-carrying recombinant Bac and their subsequent hybridization with NPs to form 
Bac-NP $\mathrm{N}_{\text {Ang1 }}$ and Bac-NP $\mathrm{Lacz}_{\text {acz }}$ were performed using protocol as mentioned in earlier work. ${ }^{13}$

\section{Detection of hAng I expressed by the transduced hASCs: enzyme- linked immunosorbent assay (ELISA) and immunofluorostaining}

In order to evaluate the transduction efficiency of Bac-NP on hASCs and compare it with free Bac and NP gene system, $\mathrm{NP}_{\text {Ang1 }}, \mathrm{Bac}_{\text {Ang1 }}$, and Bac-NP $\mathrm{N}_{\text {Ang1 }}$ at multiplicity of infection (MOI; defined as plaque forming units per cell) of 200 were used. hASCs were seeded in six-well plates at $0.5 \times 10^{6}$ cells/well and incubated overnight at $5 \%$ carbon dioxide and $37^{\circ} \mathrm{C}$. Following this, an appropriate volume of the nanocomplexes suspended in phosphate buffered saline (PBS; Life Technologies, Burlington, ON, Canada) was added to each well and incubated for 4 hours. Following this, the transduction solution was replaced with fresh media and grown in a cell culture incubator. Conditioned media were collected every alternate day for 21 days and stored at $-80^{\circ} \mathrm{C}$ for Ang 1 ELISA (R\&D Systems, Inc, Minneapolis, MN) analysis using standard procedure provided by the manufacturer. ${ }^{13}$ To detect the Ang1 expressed within the transduced cells, in another set of experiments, hASCs transduced with $\mathrm{NP}_{\text {Angl }}, \mathrm{Bac}_{\text {Angl }}$, and Bac-NP $\mathrm{NAnl}_{\text {An }}$ or nontreated controls were grown on glass microscope slides for 96 hours. After washing with PBS, the cells were fixed with $-20^{\circ} \mathrm{C}$ methanol for 10 minutes followed by immunostaining as mentioned elsewhere. ${ }^{23}$ Briefly, after blocking for 1 hour with 10\% donkey serum (Santa Cruz Biotechnology, Santa Cruz, CA), the cells were incubated overnight at $37^{\circ} \mathrm{C}$ with 1:50 dilution of goat anti-hAng1 (Santa Cruz Biotechnology) primary antibodies. On the second day, the cells were thoroughly washed with wash buffer. The cells were incubated with donkey anti-goat immunoglobulin G-tetramethyl rhodamine isothiocyanate (Santa Cruz Biotechnology) with 1:200 dilutions for 1 hour. The proportions and intensities of tetramethyl rhodamine isothiocyanate-positive hASCs, as seen under fluorescence microscope (Eclipse TE2000U; Nikon Corporation, Tokyo, Japan), gave a qualitative idea of the relative amount of cellular Ang1 expressed due to transgene delivery by the different delivery systems.

\section{Cell proliferation and viability assay}

For the cell proliferation assay, $2 \times 10^{4}$ HUVEC cells/ well were seeded in triplicate for each sample in 96-well plates. After 8 hours of culturing, the cells were washed twice with PBS and $200 \mu \mathrm{L}$ of conditioned media from nontransduced hASCs, Bac-NP Lacz -transduced hASCs,
Bac-NP ${ }_{\text {Ang1 }}$-transduced hASCs, and Bac-NP $\mathrm{N}_{\text {Ang1 }}$-transduced hASCs supplemented with anti-hAng1 antibodies were added to the corresponding set of wells. After 96 hours, absorbance was measured at $490 \mathrm{~nm}$ using CellTiter $96^{\circledR}$ AQueous Non-Radioactive Cell Proliferation Assay (Promega, Fitchburg, WI) in a Victor3 Multi Label Plate Counter (Perkin Elmer, Montreal, QC, Canada) ${ }^{24}$ In a similar way, cardiomyocyte cell viabilities under oxidative stress in groups treated with different conditioned media were measured as described later in the study, using the same assay. The experiment was performed in triplicate.

\section{Wound healing assay with HUVECs}

In order to check the wound healing potential of released Ang1, HUVECs were seeded into 24-well plates and grown to confluency. After 24 hours of serum starvation (1\% FBS), lesions were made in the monolayer using a cell scraper. ${ }^{13}$ Cells were rinsed with PBS, and then incubated with the hASC cardiomyocytes from different experimental groups $\left(\mathrm{NP}_{\mathrm{Ang}}\right.$, $\mathrm{Bac}_{\mathrm{Ang} 1}$, and $\mathrm{Bac}-\mathrm{NP}_{\mathrm{Ang} 1}$ ) for 24 hours. Cardiomyocytes from untransduced hASCs were used as control. To confirm the beneficial effect of Ang1 in particular, cardiomyocytes from Bac-NP ${ }_{\text {Ang1 }}$ group were preincubated with anti-Ang1 neutralizing antibody (R\&D Systems; $1 \mu \mathrm{g} / \mathrm{mL}$ ) for $30 \mathrm{~min}-$ utes before being added to the wells. Cells were fixed with 4\% paraformaldehyde (Sigma-Aldrich, St Louis, MO) after 24 hours and the number of cells which had moved across the starting scratched lines were measured for all groups. Three fields were analyzed for each well at $200 \times$ magnification under bright field settings of the microscope mentioned earlier.

\section{Apoptosis assay using cardiomyocytes}

H9c2 cells were seeded in 96-well microtiter plates at a density of $2 \times 10^{4}$ cells/well and cultured overnight. The media was replaced with cardiomyocytes from different treatment groups and oxidative stress was induced by adding $200 \mu \mathrm{M}$ hydrogen peroxide to the media as described elsewhere. ${ }^{25}$ After 6 hours, the apoptotic cells were detected by tracking the loss of mitochondrial membrane potential using MitoCapture ${ }^{\mathrm{TM}}$ Apoptosis Detection Kit (BioVision, Inc, Mountain View, CA) cell staining cationic dye, according to the manufacturer's protocol, which fluoresces differently in healthy and apoptotic cells under fluorescence microscope (488-nm and 543-nm excitation filters). The red emission of the dye is due to a potential-dependent aggregation in the mitochondria reflecting normal membrane potential and the green fluorescence detects the monomeric form of MitoCapture, appearing in the cytosol after mitochondrial membrane depolarization. ${ }^{26}$ For each 
experimental group, total cell number was counted and related to the number of cells that displayed fluorescence. In a similar way, after oxidative stress of 6 hours, the cell viabilities were also checked using CellTiter 96 assay as mentioned above.

\section{In vivo studies in myocardially infarcted rat model}

Immunocompetent female Lewis rats (200-250 g; Charles River Laboratories, Senneville, QC, Canada) were used. All procedures were in compliance with the Guide for the Care and Use of Laboratory Animals (National Institutes of Health publication 85-23) and the Guide to the Care and Use of Experimental Animals of the Canadian Council on Animal Care. Female Lewis rats were anesthetized. The rats were intubated with an 18-gauge catheter and mechanically ventilated (Harvard Ventilator 683; Harvard Apparatus, St Laurent, QC, Canada) at 80 breaths/minute. Anesthesia was maintained with $3 \%$ isoflurane. A left thoracotomy was performed through the fourth intercostal space to expose the left ventricle. The left coronary artery was ligated $2 \mathrm{~mm}$ from its origin with a 7-0 polypropylene suture (Ethicon, Inc, Somerville, NJ) using standard protocol. ${ }^{13}$ The ischemic myocardial segment rapidly became identifiable through its pallor. Fifteen minutes after ligation of the artery, intramyocardial injections were given. In the control group with no hASC $(n=8), 300 \mu \mathrm{L}$ of the culture medium was divided into three equal periinfarct left ventricular intramyocardial injections using a 27 gauge needle. In the hASC group $(n=8)$, the same procedure was repeated using culture medium containing $3 \times 10^{6} \mathrm{hASC}$, while the group hASCAng1 $(n=8)$ received the same number of cells transduced

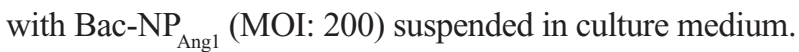

To detect the transgene expression in transplanted cells, three animals underwent the same experimental procedure as described above, followed by injection of three million cells

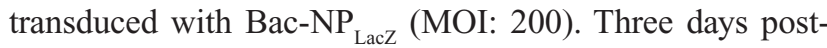
transplantation, LacZ expression was detected in histological samples using a standard staining LacZ staining procedure. ${ }^{13}$

\section{Detection of transplanted male hASCs in female rat hearts: polymerase chain reaction (PCR) analysis for human $Y$ chromosome}

In order to detect the transplanted hASCs from the male donor in heart tissue, samples from groups with no hASC $(n=6)$, hASC $(n=6)$, and hASC-Ang1 $(n=6)$ were taken. After 3 days and 28 days post-injection, three animals from each group were sacrificed and heart tissue samples were snap frozen. PCR analysis was done on the extracted DNA samples to confirm the survival of the implanted gendermismatched cells in the hearts using standard procedure followed in earlier studies. ${ }^{27}$ Briefly, equal amounts of DNA were extracted from peri-infarct portions of the ventricular heart in each group and PCR analysis was done to confirm the survival of the implanted gender-mismatched cells in the hearts 3 days and 28 days post-operation. Genomic DNA was purified using DNeasy (QIAGEN, Valencia, CA) according to the manufacturer's instructions, and the presence of living male cells in female hearts was confirmed by targeting a specific microsatellite sequence within the Y chromosome (DYS390). The primer pairs used were forward primer 5'TATATTTTACACATTTTTGGGCC3' and reverse primer 5'TGACAGTAAAATGAACACATTGC3' with product length of 250 base pairs.

\section{Scar area analysis: myocardial infarct size}

Twenty-eight days post-operation, rats were deeply anesthetized and sacrificed by rapid excision of the heart. The excised hearts were immediately soaked in cold saline to remove excess blood from the ventricles and fixed in neutral-buffered $4 \%$ formalin. Paraffin embedded samples were sectioned at $5 \mu \mathrm{m}$, and Masson's trichrome staining (Diagnostic BioSystems, Pleasanton, CA) was performed to delineate scar tissue (blue color) from the total area of myocardium. ${ }^{22,28}$ Masson's trichrome-stained section images were analyzed by ImageJ 1.41 software (National Institutes of Health, Bethesda, MA). Infarct area, epicardial and endocardial length of infarction, and ventricular and septal wall thickness were calculated and expressed as a percentage.

\section{Immunohistochemistry for detecting neovascularization}

Neovascularization was evaluated by analyzing capillary and arteriole density in the periinfarct area. For this, immunohistochemical staining was performed with anti-PECAM (Santa Cruz Biotechnology) for identification of endothelial cells and antismooth muscle $\alpha$-actin (Santa Cruz Biotechnology) for tracing the smooth muscle cells, as described elsewhere. ${ }^{22}$ Briefly, for measurement of capillary density, five fields in the periinfarct area were imaged with $200 \times$ magnification and average numbers of capillaries $<10 \mu \mathrm{m}$ in diameter were counted. Capillary density was quantified as (mean total PECAM-positive microvessels) $/ \mathrm{mm}^{2}$ using three tissue sections spanning the periinfarct tissue region of each animal. Similarly, arteriole densities were quantified as (mean total smooth muscle $\alpha$-actin-positive microvessels) $/ \mathrm{mm}^{2}$. 


\section{Transthoracic echocardiography}

Transthoracic echocardiography was performed on all surviving animals in rat groups treated with hASC $(n=8)$, hASC-Ang1 $(\mathrm{n}=8)$, and untreated control $(\mathrm{n}=8)$ on day 3 , day 14, and day 28 post-infarction. Echocardiograms were obtained with a commercially available system $\left(\operatorname{Titan}^{\circledR}\right.$; SonoSite, Inc, Bothell, WA) equipped with a $15-\mathrm{MHz}$ transducer. After sedating the animals with $2 \%$ isoflurane, echocardiography was performed according to the American Society of Echocardiology leading-edge method. ${ }^{27}$ Briefly, parasternal long- and short-axis views were obtained with both M-mode and two-dimensional images. Left ventricular end-diastolic and end-systolic diameters were measured with M-mode tracings between the anterior and posterior walls from the short-axis view just below the level of the papillary muscles of the mitral valve. Two images on average were obtained in each view and averaged over three consecutive cardiac cycles. Left ventricular end-diastolic volume was calculated as $7.0 \times$ left ventricular end-diastolic diameter $^{3} /(2.4+$ left ventricular end-diastolic diameter $)$, and left ventricular end-systolic volume as $7.0 \times$ left ventricular end-systolic diameter ${ }^{3} /(2.4+$ left ventricular end-systolic diameter). Ejection fraction was estimated as (left ventricular end-diastolic volume - left ventricular end-systolic volume)/ left ventricular end-diastolic volume.

\section{Statistical analysis}

Quantitative variables are presented as mean \pm standard deviation from independent experiments as described in the figure legends. Statistics were performed using two-way and/or one-way analysis of variance by Bonferroni's multiple comparison post hoc test. All statistical analyses were performed with GraphPad Prism (v 5.0; GraphPad Software, Inc, La Jolla, CA). $P<0.05$ was considered significant.

\section{Results}

\section{Bac-Ang I-transduced hASCs can efficiently release $\mathrm{hAng}$ I}

Released Ang1 from the transduced hASCs was analyzed at protein level by hAng1 ELISA. As shown in Figure $2 \mathrm{~A}-\mathrm{C}$, hAng1 expression was detected from day one post-transduction until day 21 with regular intervals for all groups $\left(\mathrm{NP}_{\mathrm{Ang} 1}, \mathrm{Bac}_{\mathrm{Ang} 1}\right.$, and $\left.\mathrm{Bac}-\mathrm{NP}_{\mathrm{Ang} 1}\right)$. The highest expression was seen on day four with the gene delivery systems. However, highest expression was achieved with Bac-NP ${ }_{\text {Ang1 }}$ (18.5 $\pm 1.2 \mathrm{ng} / 10^{6}$ cells), which stayed persistently higher than other groups all throughout the experimental period.
Moreover, cellular Ang1 expression, demonstrated by tetramethyl rhodamine isothiocyanate-stained cells 96 hours post-transduction, was shown to be highest in the Bac$\mathrm{NP}_{\text {Ang1 }}$-treated group as compared to $\mathrm{NP}_{\text {Ang1 }}$ and $\mathrm{Bac}_{\mathrm{Ang} 1}$ (Figure 2D-G). ELISA and immunostaining analysis confirmed that Bac- $\mathrm{NP}_{\text {Ang1 }}$ was a superior gene delivery vehicle than free $\mathrm{Bac}_{\text {Ang1 }}$ and $\mathrm{NP}_{\text {Ang1 }}$ alone, and was able to express higher amounts of hAng1 transgene at both extracellular and intracellular level.

\section{Biologic activity of the secreted hAng I on vascular endothelial cells}

To confirm the functionality of the expressed hAng1, HUVEC proliferation assay was performed using the conditioned media from Bac-NP ${ }_{\text {Ang1 }}$-transduced hASCs and compared with that of control Bac-NP $\mathrm{Lacz}^{\text {-transduced hASCs and }}$ untransduced hASCs. As reported in Figure 3A, the samples with high Ang1 concentrations, ie, from cardiomyocytes of Bac-NP ${ }_{\text {Ang1 }}$-transduced hASCs, significantly increased the proliferation rate of HUVECs $\left(2.67 \times 10^{4}\right.$ cells compared to $1.85 \times 10^{4}$ cells in Bac-NP ${ }_{\text {Lac }}$ cardiomyocytes, $1.89 \times 10^{4}$ in control nontransduced cardiomyocytes, and $1.59 \times 10^{4}$ cells in cardiomyocytes with antibody $-2 \times 10^{4}$ cells were taken as the initial density).

Furthermore, the ability of cardiomyocytes (containing secreted hAng1) to increase HUVEC wound healing in a monolayer was tested. As depicted in Figure 3B and C, stimulation of wounded HUVEC monolayer with cardiomyocytes from Bac-NP $\mathrm{Nng}_{\text {An }}(33.3 \% \pm 2.0 \%)$ induced a significant reduction of wound compared with the unstimulated control $(16.8 \% \pm 1.4 \%)$ and cardiomyocytes from Bac-NP ${ }_{\text {LacZ }}$ $(16.1 \% \pm 4.2 \%)$. Thus, consistent with mitotic assay results, Bac-NP ${ }_{\text {Ang1 }}$ showed highest healing potential, indicating that efficient overexpression of Ang1 is one of the determining factors for inducing substantial biological effects. Preincubation of cardiomyocytes with neutralizing anti-Ang1 antibodies completely inhibited Bac-NP ${ }_{\text {Ang1 }}$ cardiomyocyteinduced wound healing, clearly suggesting that chemotactic signals from hAng1 are essential for this effect.

\section{Biologic activity of the secreted hAngI on cardiomyocytes}

In order to assess whether the secreted hAng1 have a protective effect on cardiomyocytes under oxidative stress, the treated $\mathrm{H} 9 \mathrm{c} 2$ cells from different groups underwent cell viability studies after 6 hours oxidative stress using hydrogen peroxide. The data in Figure 4A show that cardiomyocytes from Bac-NP $\mathrm{Ang}_{1}$ were able to significantly reduce cell death 
A

B
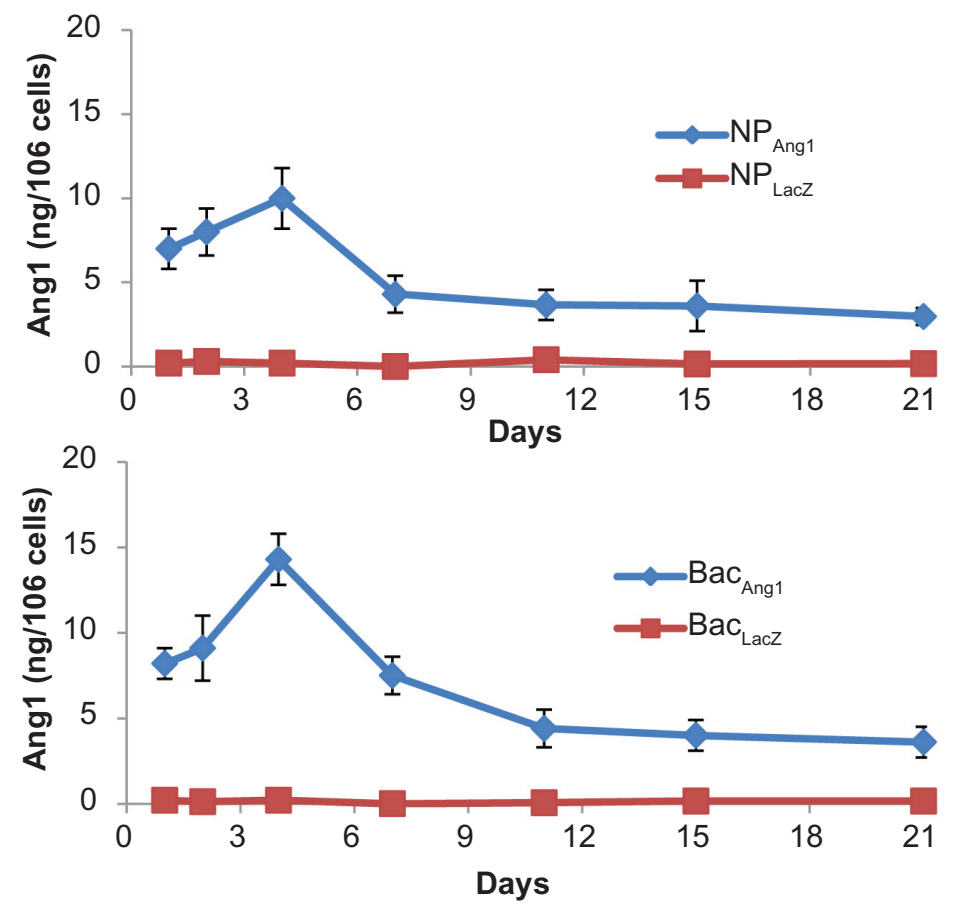

C

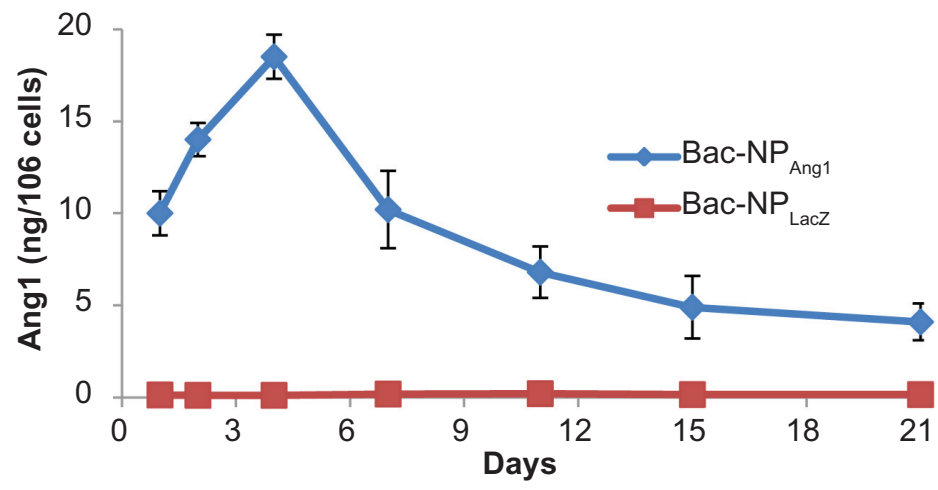

D Control untreated
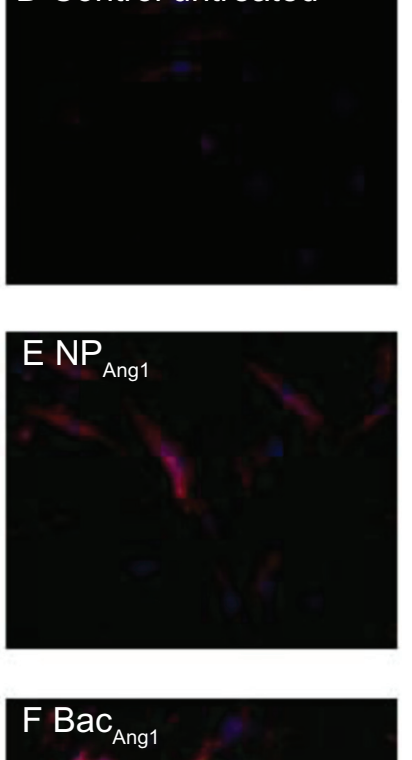

\section{G Bac-NP $_{\text {Ang1 }}$}

Figure 2 Time course profile of human angiopoietin-I expression by human adipose tissue-derived stem cells transduced with $(\mathbf{A}) \mathrm{NP}_{\text {Angl }}$, $(\mathbf{B}) \mathrm{Bac}_{\mathrm{Ang}}$, and $(\mathbf{C}) \mathrm{Bac}_{\mathrm{N}} \mathrm{NP}_{\mathrm{Ang}}$. $\mathrm{NP}_{\text {LacZ, }}, \mathrm{Bac}_{\text {LacZ }}$, and Bac-NP $\mathrm{Lacz}_{\text {, }}$ were used as control groups. Quantification of secretory human angiopoietin-I expression in the media by enzyme-linked immunosorbent assay was done at different time points after human adipose tissue-derived stem cell transduction in different media. Results are mean \pm standard deviation for three independent experiments. Fluorescent immunostaining of human adipose tissue-derived stem cells transduced with (D) untreated control, (E) $\mathrm{NP}_{\text {Angl }},(\mathbf{F}) \mathrm{Bac}_{\text {Angl }}$, and $(\mathbf{G}) \mathrm{Bac}_{-\mathrm{NP}_{\text {Angl }}}$ to detect intracellular human angiopoietin-I expression. Human angiopoietin-I expression was visualized by tetramethyl rhodamine isothiocyanate-conjugated secondary antibodies (red, tetramethyl rhodamine isothiocyanate; blue, 4',6-diamidino-2-phenylindole).

Abbreviations: AngI, angiopoietin-I; Bac $\mathrm{Ang}_{1}$, angiopoietin-I-carrying baculovirus; $\mathrm{Bac}_{\text {LacZ, LacZ-carrying baculovirus; Bac-NP }}$ Angl, angiopoietin-I-carrying baculovirus-

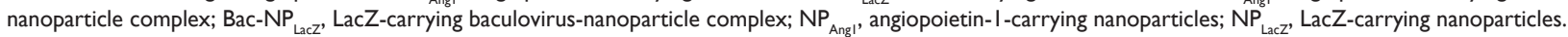

compared to Bac-NP $\mathrm{NacZ}_{\text {Lac }}$ and Bac-NP $\mathrm{NP}_{\text {Ang1+antibody }}(1.82 \pm 0.02$ versus $1.04 \pm 0.04$ versus $0.93 \pm 0.08$ ), taking a normalized value of the cell number in the untreated control group as 1.0. As positive control, cells with no oxidative stress were taken which showed a viability amount of $1.82 \pm 0.02$.

Furthermore, the antiapoptotic effect of the released hAng1 on cardiomyocytes under oxidative stress was examined and quantified by MitoCapture cell staining (Figure 4B and C). Cultivation of H9c2 cardiomyocytes under hydrogen peroxide-induced oxidative stress for 6 hours provoked a strong apoptotic response with $60.7 \% \pm 3.7 \%$ of apoptotic cells in the untransduced control cardiomyocyte group. Addition of cardiomyocytes from Bac-NP $\mathrm{Nng}_{\mathrm{Ang}} \mathrm{hASCs}$ strongly suppressed cardiomyocyte apoptosis $(24.1 \% \pm 2.9 \%)$ in comparison to other groups, which did not show any significant effect.

\section{hASCs can survive and efficiently express their transgene in vivo}

To analyze whether the xenotransplanted genetically modified hASCs were able to survive and express the transgene in the rat heart, hearts $(n=3)$ were myocardially infarcted 
A

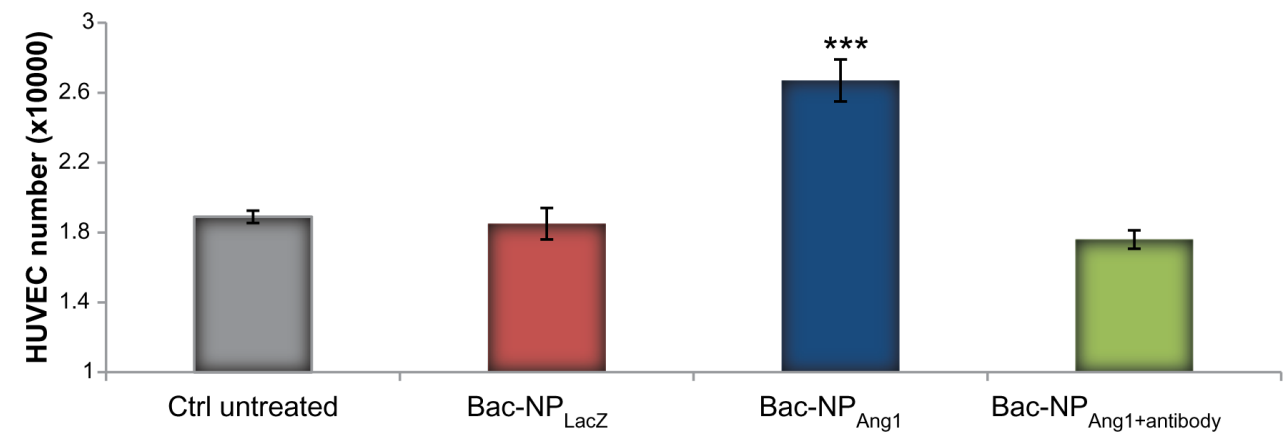

B

Conditioned media

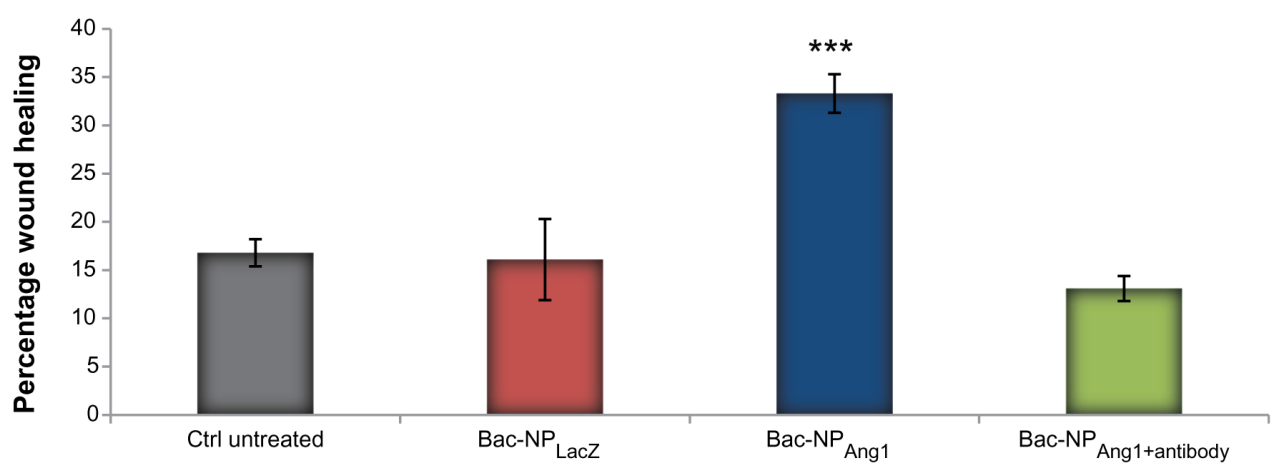

C

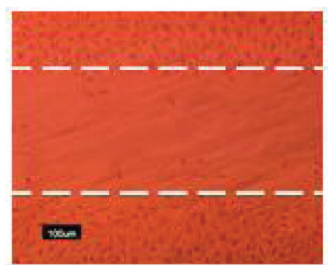

Ctrl untreated

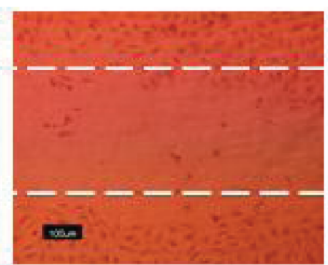

Bac-NP

Conditioned media

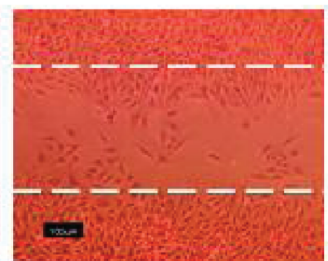

Bac-NP ${ }_{\text {Ang1 }}$

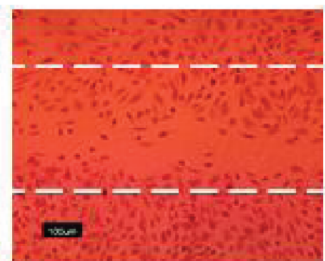

Bac-NP

Figure 3 (A) Proliferation of HUVECs grown in the presence of cardiomyocytes from Bac-NP $\mathrm{L}_{\text {Lacz }}$, Bac-NP $\mathrm{P}_{\text {Angl }}$, and nontreated control human adipose tissue-derived stem

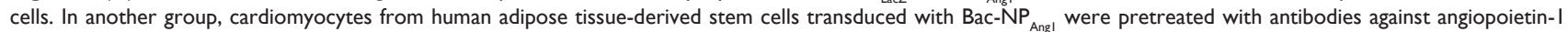
$\left(\mathrm{I} \mu \mathrm{g} / \mathrm{mL}\right.$ ). Initial seeding density was $2 \times 10^{4}$ cells/well. Proliferation was measured by a colorimetric assay after 96 hours of growth. Mean \pm standard deviation from three experiments are shown. (B) Human angiopoietin-I induces migration of endothelial cells in a wound healing assay. HUVEC monolayer was wounded with cell scraper and treated with cardiomyocytes from human adipose tissue-derived stem cells transduced with Bac-NP $P_{\text {Lacz }}$ and Bac-NP $P_{\text {Angl }}$. As another group, cardiomyocytes from Bac-NP $P_{\text {Angl }}$ were previously treated with an excess of neutralizing anti-human angiopoietin- $\mathrm{I}$ antibodies $(\mathrm{I} \mu \mathrm{g} / \mathrm{mL})$ before performing the wound healing assay. Cardiomyocytes from untreated/nontransduced cells were used as the control group. (C) HUVECs were photographed (200x) after 24 hours treatment and percentage of scratched area (which was initially free of cells, marked by the white dotted border line) covered by the migrated cells was analyzed using ImageJ I.4I software (National Institutes of Health, Bethesda, MA) as presented in (B).

Notes: Mean \pm standard deviation from three independent experiments is shown. One-way analysis of variance: statistically significant differences between groups compared to unstimulated control are indicated as $* * * P<0.001$.

Abbreviations: Bac-NP ${ }_{\text {Angl }}$, angiopoietin-I-carrying baculovirus-nanoparticle complex; Bac-NP LacZ, LacZ-carrying baculovirus-nanoparticle complex; ctrl, control; HUVEC, human umbilical vein endothelial cell.

and injected with hASCs transduced with Bac-NP $\mathrm{LacZ}_{\text {in }}$ ine left ventricular region, as described earlier. Three days posttransplantation, the rat hearts were harvested and ventricular tissue samples were stained with X-gal in order to trace the transplanted LacZ-expressing hASCs in the peri-infarct region of the heart. Figure 5A confirms that the transplanted cells were able to survive and express the transgene in the heart.

\section{Higher retention of implanted hASC-Angl in rat myocardium}

After confirming transplant survival and transgene expression, the effect of Ang1-expressing hASCs compared to normal hASCs on cardiac cellular transplant retention was further explored. Three groups (no hASC [n=6], hASC [n=6], and hASC-Ang1 $[\mathrm{n}=6])$ were taken as mentioned earlier. 
A

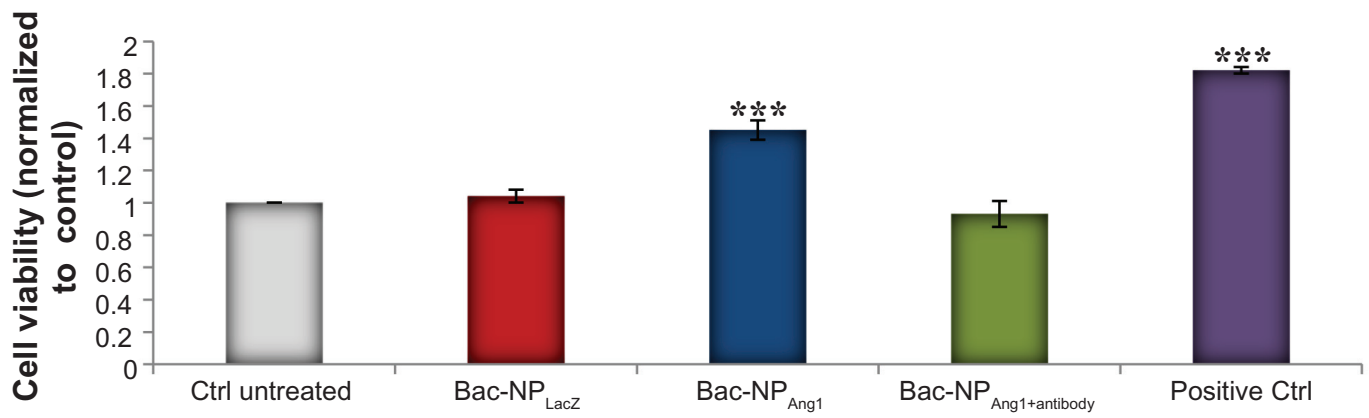

B

\section{Conditioned media}

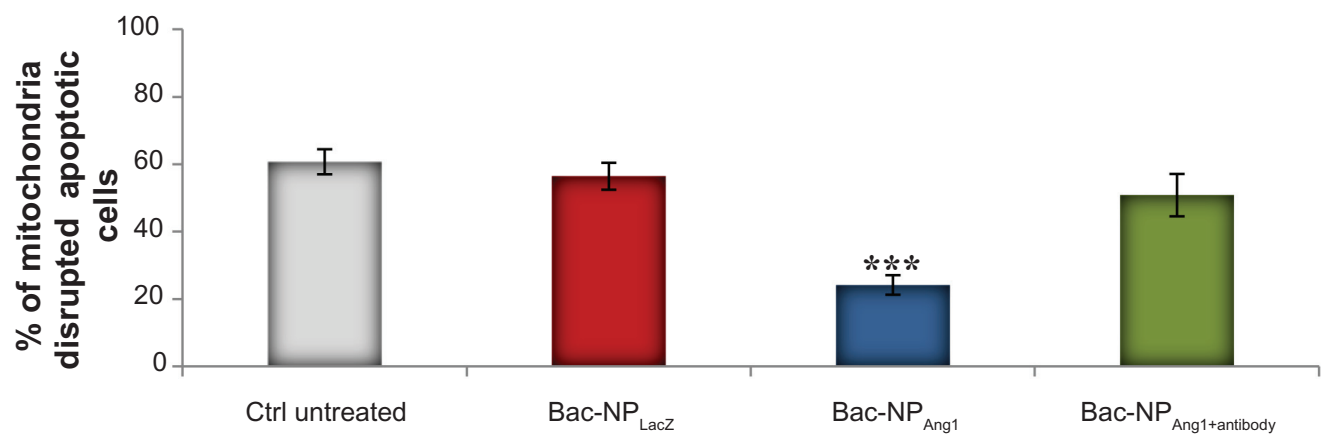

Conditioned media

C

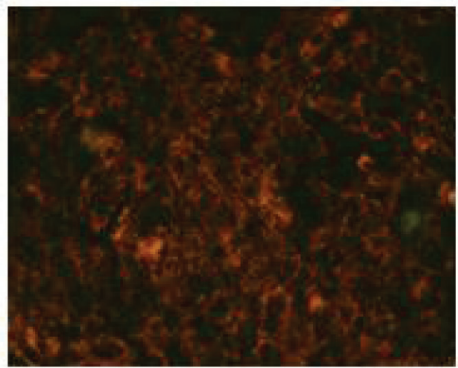

Positive control

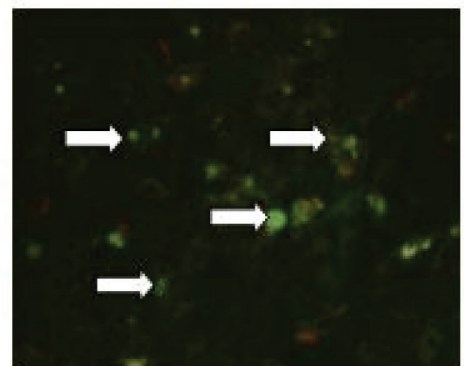

Bac-NP $_{\text {LacZ }}(\mathrm{CM})$

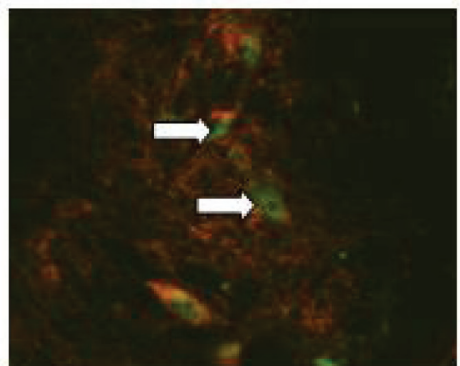

Bac-NP Ang1 $_{1}(\mathrm{CM})$

Figure 4 (A) Quantification of viable H9c2 cells when exposed to oxidative stress. H9c2 cells under different human adipose tissue-derived stem cell cardiomyocytes

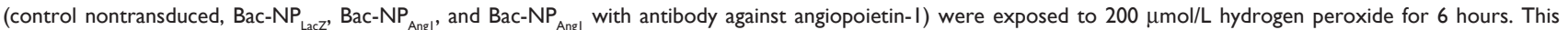
was followed by cell viability analysis. The viabilities of $\mathrm{H} 9 \mathrm{c} 2$ cells in all groups were normalized to that of control untreated. (B) Under the same conditions, quantification of $\mathrm{H} 9 \mathrm{c} 2$ cardiomyocyte apoptosis was performed by detecting the intact and disrupted mitochondrial membrane potential using MitoCapture probe. After oxidatives insult, MitoCapture staining was performed and the percentage of apoptotic cells was calculated for each group. (C) Cells with mitochondrial damage show diffuse green fluorescence, whereas cells with intact mitochondrial membrane potential exhibit red fluorescence (magnification 200x). Cells without oxidative stress were taken as positive control. Arrows indicate the apoptotic cells. Data confirm the human angiopoietin-I released from human adipose tissue-derived stem cells transduced with Bac-NPAng I are functionally active and can significantly inhibit cardiomyocyte cell death and apoptosis induced by hypoxic condition.

Notes: The graphs represent the mean \pm standard deviation from three independent experiments. Analysis of variance: statistically significant differences between groups compared to control untreated (cardiomyocytes) are indicated. $* * * P<0.00 \mathrm{I}$.

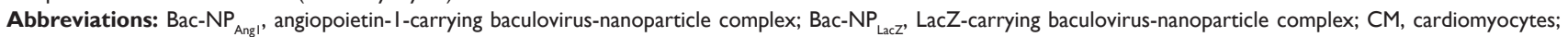
ctrl, control.

Three days and 28 days post-injection, three animals from each group were sacrificed for PCR analysis.

DNA was extracted from the injected sites of the heart tissues to detect the presence of $\mathrm{Y}$ chromosome of transplanted male hASC using standard PCR. The gel electrophoresis of the PCR products shows a clear distinction in band intensities of hASC-Ang1 and normal hASCs (Figure 5B). The hASC-Ang1 group showed around 1.72 times higher retention of viable transplanted cells in the heart in comparison to normal hASCs $(0.55$ times versus 
In vitro expression

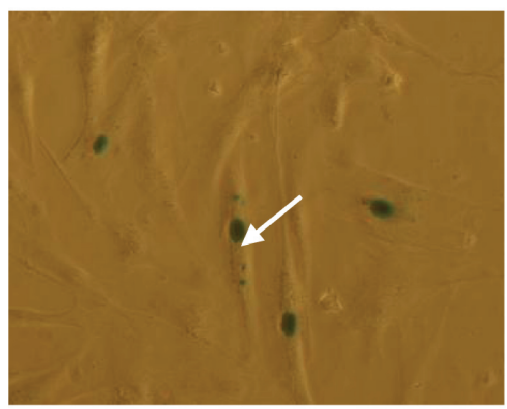

A (i)
In vivo expression

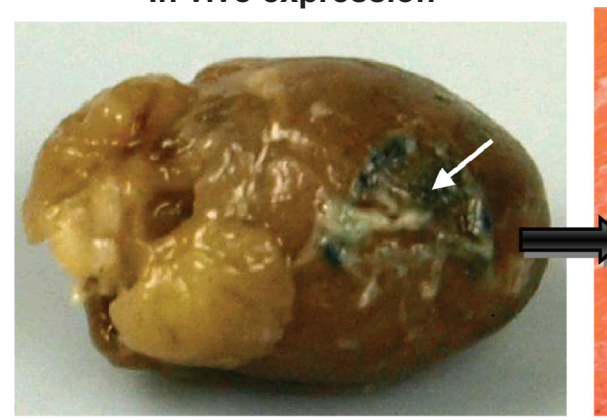

A (ii)
Histology of heart section

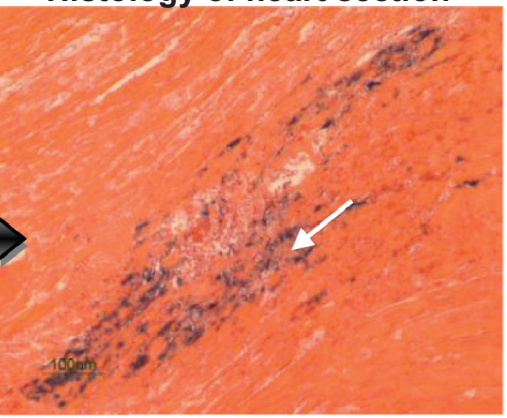

A (iii)

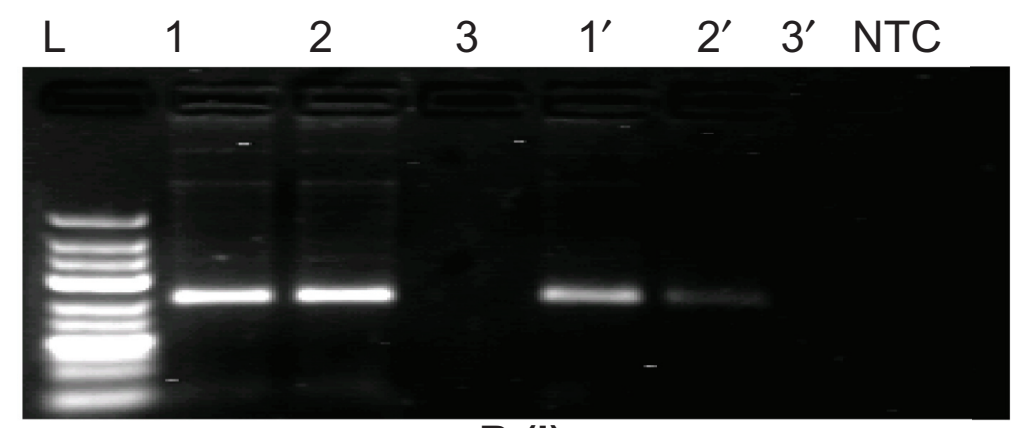

B (i)

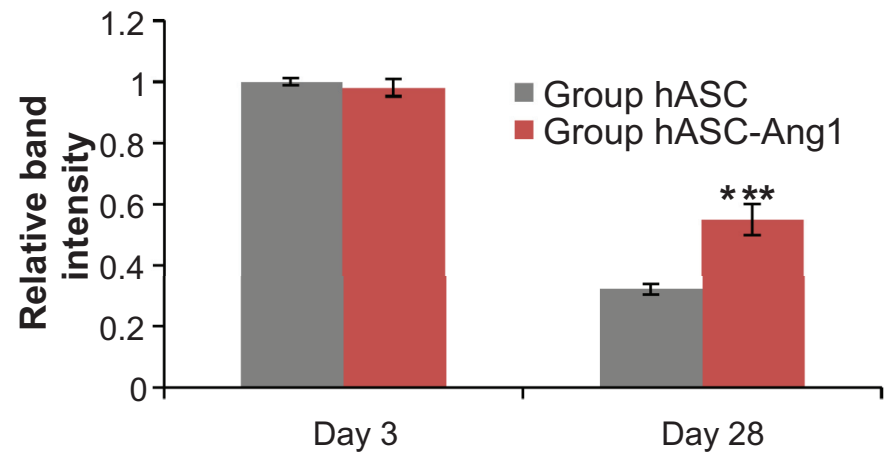

B (ii)

Figure 5 (A) Detection of transgene expression (LacZ) in baculovirus transduced hASCs transplanted in the myocardium. (i) Three million cells were transduced with Bac-NP (multiplicity of infection: 200 ) and intramyocardially injected in the left ventricular myocardium. (ii) Three days post-transplantation, hearts ( $\mathrm{n}=3$ ) were harvested and traced for LacZ expression. (iii) Histological analysis of ventricular portion (200x: stained with eosin) shows the transplanted cells expressing the LacZ. Arrows show the transplanted stained hASC-LacZ cells in the periinfarct ventricle region. (B) Higher retention of hASC-Angl compared to only hASCs in the left ventricular myocardium 28 days post-transplantation. (i) Polymerase chain reaction products ( 250 base pairs) specific for the human $Y$ chromosome (DYS390 sequence) as detected in $2 \%$ agarose gel. There were clear distinct bands in group hASC-AngI (Lane I and I') and group hASC (Lane 2 and 2') in all female rat hearts both on day three and day 28, while these bands were absent in no hASC control group (Lane 3 and $3^{\prime}$ ). Note that these band intensities were much lower in day 28 compared to day three, with group hASC having the least intensity. Representative polymerase chain reaction products from each group are shown here. (ii) ImageJ I.4I software (National Institutes of Health, Bethesda, MA) analysis of the band intensities show the hASC group has a mean of 0.32 times $(n=3)$ and group hASC-Angl has a mean of 0.55 times $(n=3)$ band intensities, taking the band intensity of group hASC on day three as 1.0 .

Notes: Two-way analysis of variance: $\mathrm{F}=34.86, P=0.0004$ (treatment groups); $\mathrm{F}=974.0, P<0.000 \mathrm{I}$ (day); $\mathrm{F}=49.4 \mathrm{I}, P=0.000 \mathrm{I}$ (interaction); $* * * P<0.00 \mathrm{I}$ versus day-matched control hASC.

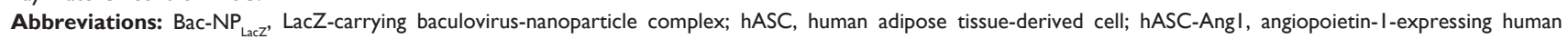
adipose tissue-derived cell; hASC-LacZ, LacZ-expressing human adipose tissue-derived cell; NTC, no template control for the polymerase chain reaction experiment.

0.32 times, taking initial 3 -day band intensity as 1.0 ; $P<0.05)$ as quantified by relative band intensities using Image J software. This semiquantitative analysis indicates that a much higher number of cells were able to survive the transplantation when they were genetically modified to express Ang1 protein.

\section{Bac-AngI hASCs can significantly attenuate scar area formation in infarcted heart}

Macroscopic views of Masson's trichrome stained heart sections are shown in Figure 6A. In both the control and cell treated groups, positively stained fibrous infarct areas were clearly observed in the heart 28 days after myocardial infarction. 
A

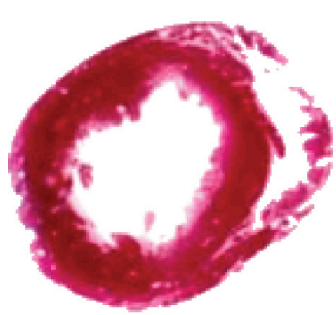

i. Sham

B

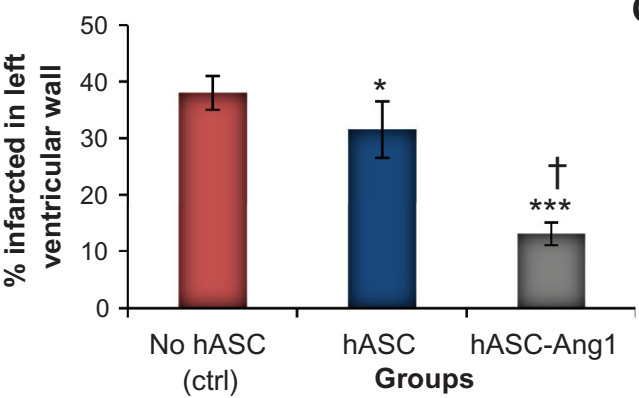

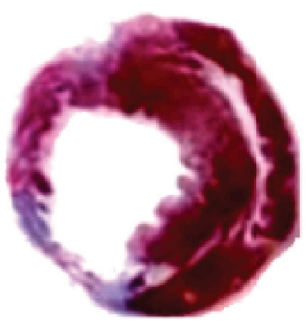

ii. No hASC (ctrl)

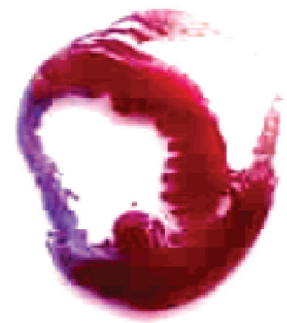

iii. hASC

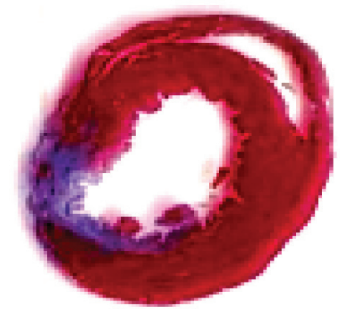

iv. hASC-Ang1

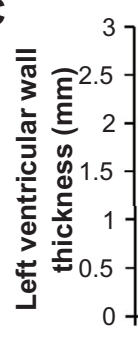

$-$

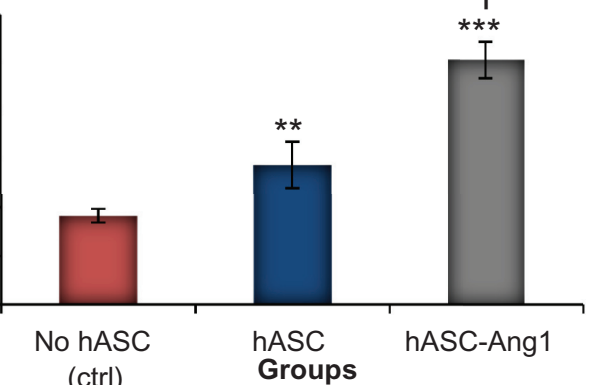

(ctrl)

Figure 6 (A) Histomorphometric analysis of infarct ventricular scar area. (i-iv) Representative images of left ventricle myocardial sections stained with Masson's trichrome show the markedly decreased cardiac fibrosis after hASC and hASC-Angl transplantations 28 days post-infarction compared to the control. The blue area represents extracellular matrix deposition in the scar tissue and the red area represents the myocardium. (B) Semiquantitative analysis of the images of the stained collagen fractions in the infarct region, using ImageJ I.4I software (National Institutes of Health, Bethesda, MA), show that hASC and hASC-AngI transplantations significantly decreased cardiac fibrosis compared to untreated group $(n=8)$ in terms of percent infarction size in left ventricular wall. (C) Moreover, hASC and hASC-AngI groups have significantly thicker left ventricular walls than untreated group. In both analyses, hASC-AngI treatment proved to be significantly better than hASC treatment.

Notes: Data expressed as mean \pm standard deviation. One-way analysis of variance: (B) $\mathrm{F}=10 \mathrm{I} .3, P<0.000 \mathrm{I}$ and $(\mathbf{C}) \mathrm{F}=\mathrm{I} 66 . \mathrm{I}, P<0.000 \mathrm{I}$ (treatment groups). Statistically significant differences between groups compared to control no hASC are indicated as $* * * P<0.00 \mathrm{I}$; $* * P<0.0 \mathrm{I}$; $* P<0.05$. Significant difference between hASC and hASCAngl is indicated by ${ }^{\dagger} P<0.00 \mathrm{I}$.

Abbreviations: hASC, human adipose tissue-derived cell; hASC-AngI, angiopoietin-I-expressing human adipose tissue-derived cell; ctrl, control.

Thin infarcts and left ventricular wall with dilated left ventricular cavity were observed in the control hearts. On the other hand, the hearts in the cell treated groups had significantly lesser infarct areas (Figure 6B: 13.0\% $\pm 3.1 \%$ for hASC-Ang1, $31.5 \% \pm 5.0 \%$ for hASCs, and $38.0 \% \pm 3.3 \%$ for control; $P<0.01$ ) and higher left ventricular wall thickness (Figure 6C: $2.53 \pm 0.19 \mathrm{~mm}$ for hASC-Ang1, $1.44 \pm 0.24 \mathrm{~mm}$ for hASCs, and $0.91 \pm 0.07 \mathrm{~mm}$ for control; $P<0.001$ ) than the control heart. A total of 24 rats were analyzed for the determination of infarct size (no hASC control [ $\mathrm{n}=8]$, hASCs [ $\mathrm{n}=8]$, and hASC-Ang1 $[\mathrm{n}=8])$. Infarct size and wall thickness in the left ventricles at the section of the middle point between ligation and apex were measured as described elsewhere. ${ }^{22}$ There was also a significant improvement in the group treated with hASC-Ang1 compared to hASCs group $(P<0.001)$ with respect to the percentage left ventricle infarct area and left ventricle infarct wall thickness.

\section{Ang I-expressing hASCs induce angiogenesis and arteriogenesis}

Reporting the significant attenuation of scar area with Ang1expressing hASCs in the last section, attempts were made to understand whether it was only the paracrine effect of the hASCs or a combinatorial effect of hASC-released Ang1 and paracrine factors which was responsible for the improvement. For this, the neovasculature formation in the periinfarct area was assessed by detecting angiogenesis and arteriogenesis densities (Figure 7). Indeed, a significant improvement was seen in angiogenesis in the hASC and hASC-Ang1 groups compared to the control $\left(201 \pm 25 / \mathrm{mm}^{2}\right.$ for hASC-Ang1, $174 \pm 14 / \mathrm{mm}^{2}$ for $\mathrm{hASCs}$, and $141.5 \pm 6 / \mathrm{mm}^{2}$ for control; $P<0.01)$. Moreover, the hASC-Ang1 group showed significantly higher capillary density compared to hASC. Similar results were obtained with arteriole density in the hASCAng1 group $\left(12.25 \pm 2.4 / \mathrm{mm}^{2}\right.$ for hASC-Ang1, $9.5 \pm 1.8 / \mathrm{mm}^{2}$ for hASCs, and $6.5 \pm 1.32 / \mathrm{mm}^{2}$ for control; $P<0.05$ ), with the hASC-Ang1 group showing significantly higher arteriole density compared to hASC.

\section{Significantly improved cardiac function is detected in hASC-Angl-treated infarcted heart}

To investigate if the reduction of scar area after stem cell therapy results in improved heart function, the ejection 

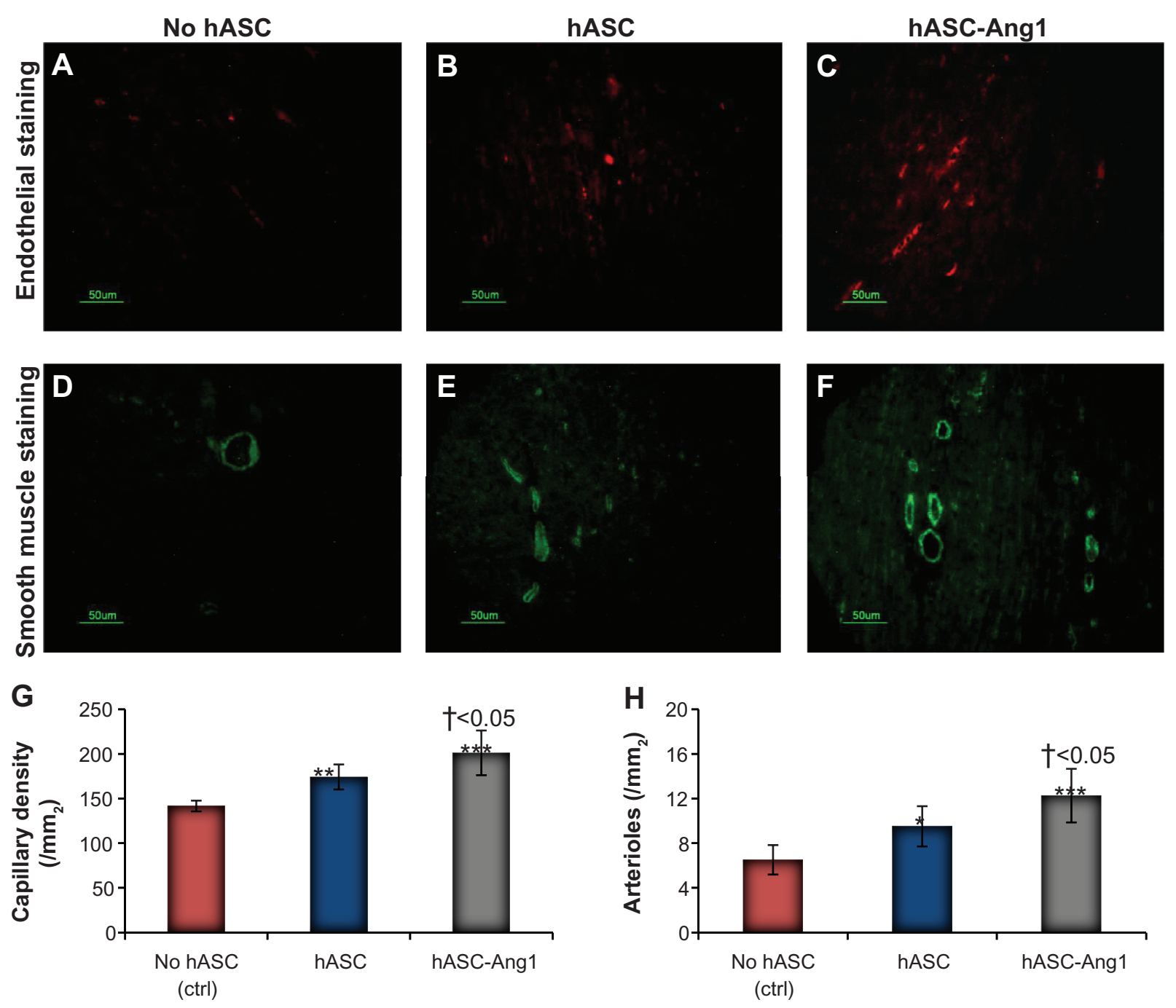

Figure 7 Angiogenesis and arteriogenesis in the periinfarct area. Immunohistological staining of CD3I to detect endothelial cells in (A) no hASC, (B) hASC, and (C) hASC-AngI groups. Immunohistological staining of smooth muscle $\alpha$-actin for smooth muscle cells in (D) no hASC, (E) hASC, and (F) hASC-Angl groups. Quantification of (G) capillary and $(\mathbf{H})$ arteriole density.

Notes: Data are expressed as mean \pm standard deviation. One-way analysis of variance: $(\mathbf{G}) \mathrm{F}=24.86, P<0.000 \mathrm{I}$ and $(\mathbf{H}) \mathrm{F}=18.48, P<0.000 \mathrm{I}$ (treatment groups). Statistically significant differences between groups compared to control no hASC are indicated as $* * * P<0.001 ; * * P<0.01$; $* P<0.05$. Significant difference between hASC and hASC-Angl is indicated by ${ }^{\dagger}$.

Abbreviations: hASC, human adipose tissue-derived cell; hASC-AngI, angiopoietin-I-expressing human adipose tissue-derived cell; ctrl, control.

fraction in a rat model of myocardial infarction at different time periods (days three, 14, and 28) was monitored. Ejection fraction of all groups was around $30 \%$ on day three post-infarction, indicating successful acute myocardial infarction in all groups. ${ }^{27}$ As presented in Figure 8, there were significant improvements in ejection fraction in groups treated with hASC-Ang1 and hASCs compared to the control group on day 14 and day 28 , although there were no significant differences between the groups on day three post-infarction $(P>0.05)$. In addition, the hASC-Ang1 treated group showed a significantly greater increase in ejection fraction as compared to hASC-treated groups on day 14 $(44.78 \% \pm 6.14 \%$ versus $37.1 \% \pm 4.9 \% ; P<0.01)$ and day
$28(46.28 \% \pm 6.3 \%$ versus $36.35 \% \pm 5.77 \% ; P<0.01)$. Thus, analysis using echocardiographic ejection fraction data suggests that hASC-Ang 1 can be a better alternative to hASCs to improve cardiac function after acute myocardial damage.

\section{Discussion}

Cardiovascular and ischemic heart disease is a major health concern in both developing and industrialized countries. Although different gene therapy approaches are being employed to treat the disease, clinical trials showed limited results suggesting the need for a better gene therapy strategy. ${ }^{29}$ It has been demonstrated in small animals, that ASCs induce wound healing effects in damaged ischemic 
A
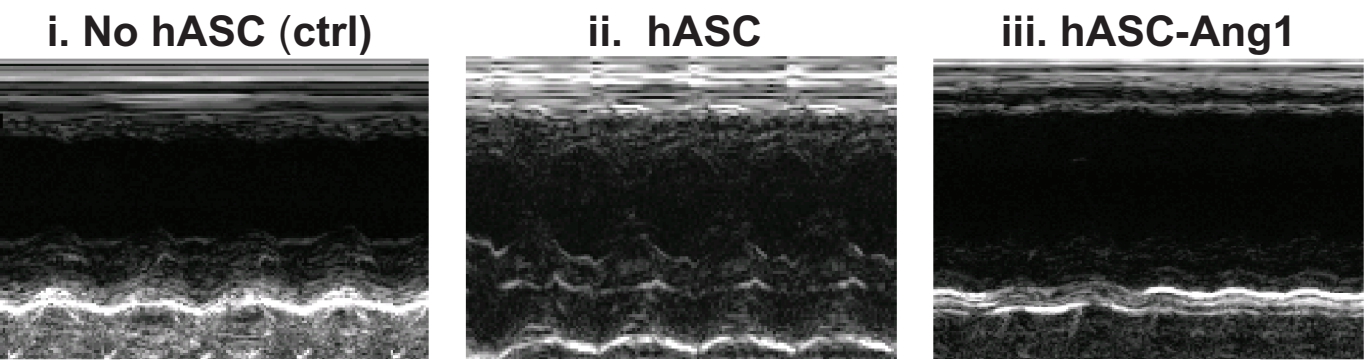

B

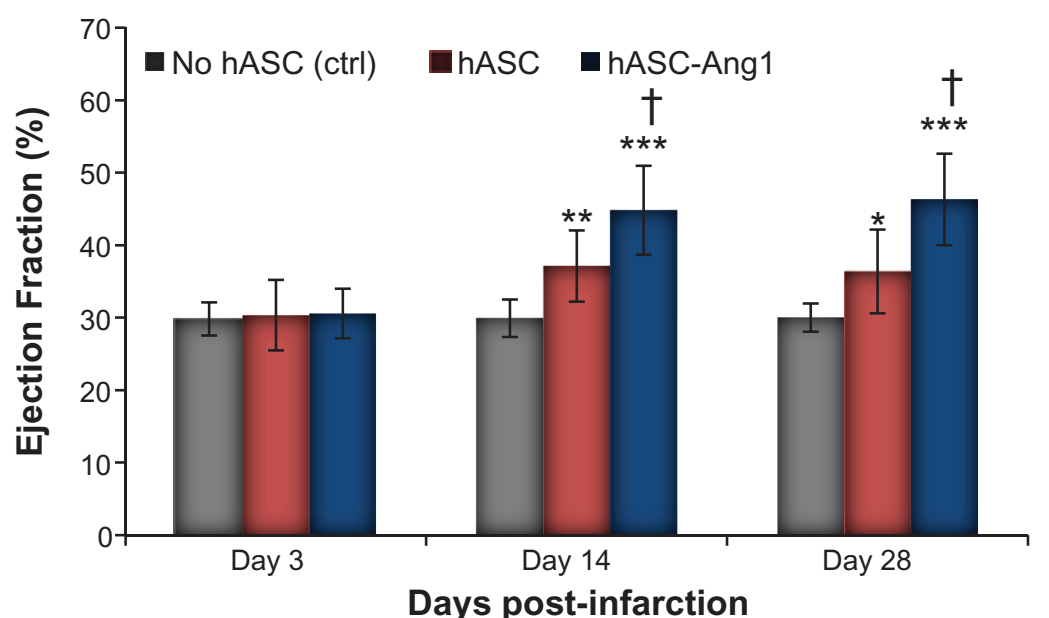

Figure 8 Echocardiographic assessment of cardiac function. Effect of hASC-Angl transplantation on cardiac function was analyzed over 28 days post-infarction. Heart ejection fraction was monitored on days three, 14 , and 28 after transplantation of medium $(n=8)$, hASCs $(n=8)$, and hASC-Angl ( $n=8)$ in rat myocardial infarction model using M-mode echocardiograms (i-iii represents data of day 28). (B) As demonstrated in the graph, ejection fraction increased significantly after treatment in the hASC-Angl and hASC groups over the 28 days post-infarction, but the untreated control group did not show any sign of improvement. Ejection fraction was significantly higher in the treated groups compared to the control on days 14 and 28 after treatment. Furthermore, hASC-Ang I group showed a significantly better ejection fraction than hASC group with time.

Notes: Mean ejection fraction \pm standard deviation is shown in graph. Echocardiogram analysis two-way analysis of variance: $F=13.28, P=0.0002$ (treatment groups); $\mathrm{F}=77.95, P<0.000 \mathrm{I}$ (time); $\mathrm{F}=28.47, P<0.000$ I (interaction); $\mathrm{F}=9.7 \mathrm{I} 6, P<0.000 \mathrm{I}$ (subjects matching); $* * * P<0.00 \mathrm{I} ; * * P<0.01 ; * P<0.05$ versus time-matched control. tP $<0.01$ versus time-matched hASC.

Abbreviations: hASC, human adipose tissue-derived cell; hASC-AngI, angiopoietin-I-expressing human adipose tissue-derived cell; ctrl, control.

tissues mainly through proangiogenic paracrine mechanism followed by neovascularization, local recruitment of progenitor cells, and exhibition of cardiomyocyte-like properties. . $^{17,30}$ Moreover, ASCs secrete VEGF which has a favorable impact on myocardial neovascularization, tissue perfusion, and contractile performance. In addition, a larger number of ASCs can be easily harvested using noninvasive standard fat extraction techniques, which is of immense importance under clinical settings because of their ready availability. ${ }^{14,18}$ Most importantly, as allogeneic hASCs can be easily obtained from young healthy donors, they could be of great value in elderly patients with advanced diseases, where their own ASCs could be dysfunctional.

The initial step involved the development of a recombinant Bac carrying Ang1 and assessing its potential for direct gene therapy for efficient myocardial neoangiogenesis as reported in previous work. ${ }^{13}$ As the next step, the present study further explored its potential for stem cell-based gene therapy, apart from direct gene therapy. The present study reports for the first time, the angiogenic potential of this newly formulated hybrid nanodelivery system composed of vascular gene-carrying recombinant $\mathrm{Bac}$ armed with noncovalently conjugated TAT/DNA NPs for stem cell-based gene therapy. As stem cells are known to have a natural potential for cardiovascular therapies, complementing them with rapid Ang1 overexpression can further improve their efficacy. The present work illustrates this hypothesis in a rat model with acute myocardial infarction. Moreover, this is the first study to report the potential of recombinant Bac to genetically modify ASCs. In addition, the work also demonstrates the potential of genetically modified ASCs for successful xenotransplantation applications.

Using a hybrid model of TAT/DNA Ang1 $_{\text {NPs coupled to }}$ $\mathrm{Bac}_{\text {Ang1 }}$ for ASC transduction under optimized conditions (Figure S1), significantly higher gene transfer efficiency and gene carrying capacity was achieved than free $\mathrm{Bac}_{\mathrm{Ang} 1}$ or $\mathrm{NP}_{\mathrm{Ang} 1}$ 
alone (Figure 2 and Figure S2). Figure S2 demonstrates that Bac-NP, with Bac MOI of 200 and N/P ratio (defined as the molar ratio of amino groups/phosphate groups) of 3 of TAT/ DNA NP, was optimal so as to achieve the highest transduction efficiency with no cytotoxic effects (Figure S3). Data also confirm that gene transfer efficiency can be significantly enhanced by this hybrid nanoformulation of Bac and NP, as compared to their individual efficiencies. In vitro analysis showed that Bac-NP-transduced cells were able to express their transgene for at least 3 weeks (Figure 2C), which is comparable to other experimental results. ${ }^{24,31}$ This temporal expression of the delivery system is beneficial in many cases, particularly for angiogenesis where the expression of the therapeutic protein ceases once its job is done. Further studies need to be done to comprehend the exact mechanism of such a gene delivery system. Rapid attachment of cationic NPs to a negatively charged cell surface followed by efficient intracellular gene entry to the nucleus by Bac may be a probable way. Optimizing the $\mathrm{pH}$ condition and transduction time may further enhance the gene delivery efficiency of the Bac-NP system. ${ }^{10}$

Bac surface modification by chemical coupling and electrostatic interactions is recently being exploited in different ways for enhanced gene delivery. ${ }^{32}$ It has been demonstrated that galactosylated polyethylenimine/DNA hybridized Bac improves gene delivery to hepatoblastoma and lung carcinoma cells. ${ }^{33}$ Coating of Bac using positively charged polymer polyethylenimine is also shown to significantly enhance their in vivo gene therapy potential compared to uncoated viruses. ${ }^{34}$ Recently Chen et al demonstrated that genetically modified Bac generated by fusing the protein transduction domain of human immunodeficiency virus TAT protein with VP39, the major capsid protein, resulted in improved transduction of various mammalian cells. ${ }^{35}$ Similarly, the present study demonstrates that surface modifying Bac with TAT/ DNA NPs can also improve gene transfer efficiency. But, to achieve significant therapeutic effects, specifically with stem cells, maintenance of their natural functionalities is of immense importance. The present study confirmed this by successfully differentiating the genetically modified hASCs to adipogenic and osteogenic lineages (Figure S4). In addition, data revealed that Bac-NP-mediated hAng1 expression from hASCs were biologically active as indicated by their enhanced mitotic and chemotactic activities on HUVEC (Figure 3). The study also confirmed that the released Ang1 possesses antiapoptotic and protective effects on cardiomyocytes (Figure 4). This supports the findings reported by Wang et al who reported that Ang1 can protect cardiomyocytes from oxidative stress-induced apoptosis. ${ }^{25}$
In addition to efficient transgene expression, retention of viability of transplanted cells at the infarct site is an important parameter that determines the myocardial therapeutic index of the study. This is because higher retention of viable cells directly correlates with enhanced secretion of paracrine factors and better scope for cellular cardiomyoplasty. Previous works have aimed to address this problem using polymeric microcapsules to reduce the biologic and mechanical loss of implanted cells in the continuously beating heart. ${ }^{11,14,36}$ In an interesting study, Liu et al demonstrated that Ang1 can protect MSC against serum deprivation and hypoxia-induced apoptosis. ${ }^{37}$ Detection of a significantly higher amount of human $\mathrm{Y}$ chromosome-positive hASCs in recipient female rat hearts treated with hASC-Ang1 compared to hASC, 28 days post-implantation, confirmed these findings (Figure 5B).

Most importantly, hASC-Ang1 treatment resulted in enhancement of vascular density (Figure 7), thereby significantly improving cardiac function (Figure 8) compared to hASC and control groups; although cardiomyogenic transdifferentiation of grafted cells was not detected in any of the groups. ${ }^{6}$ Moreover, histological findings confirmed the reduction of fibrosis in the infarct area (Figure 6), which is an important indicator for improved heart function since late reperfusion of infarcted vascular beds attenuates left ventricular remodeling including infarct expansion. ${ }^{4}$ Measurement of blood flow using cardiac magnetic resonance imaging-based techniques can give a better real time picture on the progress of such myocardial regenerative process post cell transplantation. Although preclinical findings exemplify the beneficial effects of such combined adipose stem cell-gene therapy treatment, further in vivo studies are needed to elucidate the precise mechanism and to investigate its prolonged effects on cardiac function; these will help determine whether one-time cell delivery is a viable option or whether multiple cell transplantations are required to achieve a long term desirable outcome.

\section{Conclusion}

From a clinical standpoint, the study promises major advancements where ASCs could be easily harvested and mass produced well in advance, genetically modified to express biologically active therapeutic proteins, and stored for immediate off-the-shelf use on any patient without delay after an acute myocardial infarction.

In summary, it was demonstrated that a hybrid nanodelivery complex of Bac and NPs can take advantage of the unique features associated with the two individual vector systems and exhibit enhanced gene transfer efficiency, supported by in vitro and in vivo experimental demonstrations. It was illustrated that 
genetically-engineered hASCs, using a hybrid nanocomplex, enhanced the transplant retention in a myocardially infarcted rat model and induced a significant favorable impact on tissue perfusion and contractile performance, which have been corroborated with vasculogenesis. Taken together, the current findings confirm the feasibility of a combined stem cell-based gene therapy for ischemic heart diseases using a novel, biologically safe nanobiohybrid gene delivery system.

\section{Acknowledgments}

The authors gratefully acknowledge the assistance received from the Canadian Institutes of Health Research (MOP \#64308) to S Prakash, and the Natural Sciences and Engineering Research Council of Canada to S Prakash and D Shum-Tim. A Paul acknowledges the Alexander Graham Bell Post Graduate Scholarship and Michael Smith Foreign Study Award - Doctoral from the Natural Sciences and Engineering Research Council of Canada.

\section{Disclosure}

The authors report no conflicts of interest in this work.

\section{References}

1. Zhang C, Sun A, Zhang S, et al. Efficacy and safety of intracoronary autologous bone marrow-derived cell transplantation in patients with acute myocardial infarction: insights from randomized controlled trials with 12 or more months follow-up. Clin Cardiol. 2010;33(6):353-360.

2. Surder D, Schwitter J, Moccetti T, et al. Cell-based therapy for myocardial repair in patients with acute myocardial infarction: rationale and study design of the SWiss multicenter Intracoronary Stem cells Study in Acute Myocardial Infarction (SWISS-AMI). Am Heart J. 2010; 160(1):58-64.

3. Atoui R, Shum-Tim D, Chiu RC. Myocardial regenerative therapy: immunologic basis for the potential "universal donor cells." Ann Thorac Surg. 2008;86(1):327-334.

4. Das H, George JC, Joseph M, et al. Stem cell therapy with overexpressed VEGF and PDGF genes improves cardiac function in a rat infarct model. PLoS One. 2009;4(1):e7325.

5. Kim SH, Moon HH, Kim HA, Hwang KC, Lee M, Choi D. Hypoxiainducible vascular endothelial growth factor-engineered mesenchymal stem cells prevent myocardial ischemic injury. Mol Ther. 2011;19(4): 741-750.

6. Chen SL, Zhu CC, Liu YQ, et al. Mesenchymal stem cells genetically modified with the angiopoietin-1 gene enhanced arteriogenesis in a porcine model of chronic myocardial ischaemia. J Int Med Res. 2009; 37(1):68-78.

7. Phillips JE, Gersbach CA, Garcia AJ. Virus-based gene therapy strategies for bone regeneration. Biomaterials. 2007;28(2):211-229.

8. Donsante A, Miller DG, Li Y, et al. AAV vector integration sites in mouse hepatocellular carcinoma. Science. 2007;317(5837):477.

9. Chuang CK, Wong TH, Hwang SM, et al. Baculovirus transduction of mesenchymal stem cells: in vitro responses and in vivo immune responses after cell transplantation. Mol Ther. 2009;17(5): 889-896.

10. Paul A, Prakash S. Baculovirus reveals a new $\mathrm{pH}$-dependent direct cell-fusion pathway for cell entry and transgene delivery. Future Virol. 2010;5(5):533-537.
11. Paul A, Shum-Tim D, Prakash S. Investigation on PEG integrated alginate-chitosan microcapsules for myocardial therapy using marrow stem cells genetically modified by recombinant baculovirus. Cardiovasc Eng Technol. 2010;1(2):154-164.

12. Lo SL, Wang S. An endosomolytic Tat peptide produced by incorporation of histidine and cysteine residues as a nonviral vector for DNA transfection. Biomaterials. 2008;29(15):2408-2414.

13. Paul A, Binsalamah ZM, Khan AA, et al. A nanobiohybrid complex of recombinant baculovirus and Tat/DNA nanoparticles for delivery of Ang-1 transgene in myocardial infarction therapy. Biomaterials. 2011;32(32):8304-8318.

14. Paul A, Cantor A, Shum-Tim D, Prakash S. Superior cell delivery features of genipin crosslinked polymeric microcapsules: preparation, in vitro characterization and pro-angiogenic applications using human adipose stem cells. Mol Biotechnol. 2011;48(2):116-127.

15. Cho KS, Park HK, Park HY, et al. IFATS collection: immunomodulatory effects of adipose tissue-derived stem cells in an allergic rhinitis mouse model. Stem Cells. 2009;27(1):259-265.

16. Duckers HJ Sr, Houtgraaf J, van Guens RJ, Fernandez-Aviles FJ, Serruys PW. First-in-man experience of adipose tissue-derived regenerative cell transplantation in the treatment of patients with an acute ST-elevation myocardial infarction (APOLLO trial). J Am Coll Cardiol. 2010;55:A100. E934.

17. Metzele R, Alt C, Bai XW, et al. Human adipose tissue-derived stem cells exhibit proliferation potential and spontaneous rhythmic contraction after fusion with neonatal rat cardiomyocytes. FASEB J. 2011;25(3):830-839.

18. Rehman J, Traktuev D, Li JL, et al. Secretion of angiogenic and antiapoptotic factors by human adipose stromal cells. Circulation. 2004;109(10):1292-1298

19. Tao Z, Chen B, Tan X, et al. Coexpression of VEGF and angiopoietin-1 promotes angiogenesis and cardiomyocyte proliferation reduces apoptosis in porcine myocardial infarction (MI) heart. Proc Natl Acad Sci U S A. 2011;108(5):2064-2069.

20. Zhou L, Ma W, Yang Z, et al. VEGF165 and angiopoietin-1 decreased myocardium infarct size through phosphatidylinositol-3 kinase and Bcl-2 pathways. Gene Ther. 2005;12(3):196-202.

21. Samuel SM, Akita Y, Paul D, et al. Coadministration of adenoviral vascular endothelial growth factor and angiopoietin-1 enhances vascularization and reduces ventricular remodeling in the infarcted myocardium of type 1 diabetic rats. Diabetes. 2010;59(1):51-60.

22. Takahashi K, Ito Y, Morikawa M, et al. Adenoviral-delivered angiopoietin-1 reduces the infarction and attenuates the progression of cardiac dysfunction in the rat model of acute myocardial infarction. Mol Ther. 2003;8(4):584-592.

23. Ye L, Haider HK, Jiang S, Ge R, Law PK, Sim EK. In vitro functional assessment of human skeletal myoblasts after transduction with adenoviral bicistronic vector carrying human VEGF165 and angiopoietin-1. $J$ Heart Lung Transplant. 2005;24(9):1393-1402.

24. Paul A, Khan AA, Shum-Tim D, Prakash S. BacMam virus transduced cardiomyoblasts can be used for myocardial transplantation using APPEG-A microcapsules: molecular cloning, preparation, and in vitro analysis. J Biomed Biotechnol. 2010;2010:858094.

25. Wang Z, Cui M, Sun L, et al. Angiopoietin-1 protects $\mathrm{H} 9 \mathrm{c} 2$ cells from $\mathrm{H} 2 \mathrm{O} 2$-induced apoptosis through AKT signaling. Biochem Biophys Res Commun. 2007;359(3):685-690.

26. Li J, Niu XL, Madamanchi NR. Leukocyte antigen-related protein tyrosine phosphatase negatively regulates hydrogen peroxide-induced vascular smooth muscle cell apoptosis. J Biol Chem. 2008;283(49): 34260-34272.

27. Atoui R, Asenjo JF, Duong M, Chen G, Chiu RC, Shum-Tim D. Marrow stromal cells as universal donor cells for myocardial regenerative therapy: their unique immune tolerance. Ann Thorac Surg. 2008;85(2):571-579.

28. Paul A, Srivastava S, Chen G, Shum-Tim D, Prakash S. Functional assessment of adipose stem cells for xenotransplantation using myocardial infarction immunocompetent models: comparison with bone marrow stem cells. Cell Biochem Biophys. 2011. [Epub ahead of print.] doi: 10.1007/s12013-011-9323-0. 
29. Stewart DJ, Kutryk MJ, Fitchett D, et al. VEGF gene therapy fails to improve perfusion of ischemic myocardium in patients with advanced coronary disease: results of the NORTHERN trial. Mol Ther. 2009; 17(6):1109-1115.

30. Cai L, Johnstone BH, Cook TG, et al. IFATS collection: human adipose tissue-derived stem cells induce angiogenesis and nerve sprouting following myocardial infarction, in conjunction with potent preservation of cardiac function. Stem Cells. 2009;27(1):230-237.

31. Jardin BA, Zhao Y, Selvaraj M, et al. Expression of SEAP (secreted alkaline phosphatase) by baculovirus mediated transduction of HEK 293 cells in a hollow fiber bioreactor system. J Biotechnol. 2008;135(3):272-280.

32. Chen CY, Lin CY, Chen GY, Hu YC. Baculovirus as a gene delivery vector: recent understandings of molecular alterations in transduced cells and latest applications. Biotechnol Adv. 2011;29(6):618-631.
33. Kim YK, Choi JY, Jiang HL, et al. Hybrid of baculovirus and galactosylated PEI for efficient gene carrier. Virology. 2009;387(1):89-97.

34. Yang Y, Lo SL, Yang J, et al. Polyethylenimine coating to produce serum-resistant baculoviral vectors for in vivo gene delivery. Biomaterials. 2009;30(29):5767-5774.

35. Chen HZ, Wu CP, Chao YC, Liu CY. Membrane penetrating peptides greatly enhance baculovirus transduction efficiency into mammalian cells. Biochem Biophys Res Commun. 2011;405(2):297-302.

36. Paul A, Ge Y, Prakash S, Shum-Tim D. Microencapsulated stem cells for tissue repairing: implications in cell-based myocardial therapy. Regen Med. 2009;4(5):733-745.

37. Liu XB, Jiang J, Gui C, Hu XY, Xiang MX, Wang JA. Angiopoietin-1 protects mesenchymal stem cells against serum deprivation and hypoxia-induced apoptosis through the PI3K/Akt pathway. Acta Pharmacol Sin. 2008;29(7):815-822. 


\section{Supplementary information Characterization of baculovirus (Bac)- nanoparticle (NP) hybrid complex}

The particle size and zeta potential of the NP, Bac, and Bac-NP hybrid particles were measured by the technique of electrophoretic laser Doppler anemometry using a Zeta Potential Analyzer (ZetaPlus; Brookhaven Instruments Corporation, Holtsville, NY). ZetaPlus Particle Sizer Software (v 4.11; Brookhaven) was used to determine the size distribution of the particles and Zeta Potential Analyzer software (v 3.57; Brookhaven) was used for zeta potential analysis. Both particle size and zeta potential were measured for three independent preparations and each measurement was obtained after taking the average of the three runs. Transmission electron microscopy (TEM) was used to obtain the size characterization. The NPs were suspended in $1 \times$ phosphate buffered saline and analyzed on CM200 FEGTEM (Royal Philips Electronics, Markham, ON, Canada). Results are presented in Figure S1.

The scanning electron microscope (SEM; S4700 FEGSEM Hitachi, Oakville, ON, Canada) and atomic force microscope (AFM; Digital Instruments, Palo Alto, CA) photomicrographs in Figure 2A and B confirm the formation of NP by transactivating transcriptional activator (TAT) and DNA complexation. The formed NPs were further studied by TEM and zeta potential analyzer, as demonstrated in Figure S1C-F. The Bac-NP complex was first characterized by measuring the zeta potential of the nanocomplex with laser Doppler electrophoresis (Figure S1C). At its natural $\mathrm{pH} 6.8$, Bac is negatively charged with zeta potential of $-6.5 \pm 1.4 \mathrm{mV}$. At physiological $\mathrm{pH} 7.4$, Bac had a zeta potential of $-12.8 \pm 3.1 \mathrm{mV}$. On the other hand, prepared TAT/DNA NPs showed high positive charge of $26.5 \pm 3.2 \mathrm{mV}$ at N/P ratio of 3 . The positively charged TAT/DNA NPs, upon conjugation with the negatively charged Bac, formed positively charged $(5.1 \pm 2.7 \mathrm{mV})$ Bac-NP hybrid nanocomplexes.

To reconfirm the successful formation of the Bac-NP complexes, particle sizes of each complex were measured. Free Bac had an average size of $238 \pm 10 \mathrm{~nm}$, whereas free TAT/DNA NP showed an average size of around $72 \pm 4.6 \mathrm{~nm}$. The Bac-NP hybrid complexes had an average size of $480 \pm 18.2 \mathrm{~nm}$. This significant increase in size of Bac-NP particles, compared to that of free Bac and NPs, indicates the efficient production of the nanobiohybrid complexes, generated by strong electrostatic interactions of Bac with the NPs.

In order to look for the morphological evidence for successful conjugation of the budded Bac particles with the NPs, TEM was used. Electron micrographs showed the well-dispersed NPs (Figure S1D). On coming in contact with free Bac (Figure S1E; the rod-shaped particles with a length of 200-250 nm), there was an instant virus-NP complex formation by the negatively charged Bac with the positively charged NP, as indicated in Figure S1F to form the hybridized Bac-NP complex. The images of Bac-NP complexes also confirm the proper retention of the typical rod-shaped morphological appearance and envelope structure of Bac, suggesting that Bac were able to sustain their morphological integrity even after hybridization with NPs.

\section{Optimization of viral dose for Bac-NP mediated human adipose tissue-derived stem cell (hASC) transduction}

In order to achieve maximum transduction, the effect of multiplicity of infection (defined as plaque forming units per cell) and their combinatorial effects on the hybrid Bac-NP system were optimized. For this, multiplicity of infection ranging from 100 to 400 and N/P ratio of 3 were used. Initially, hASCs were seeded in six-well plates at $0.5 \times 10^{6}$ cells/well and incubated overnight at $5 \%$ carbon dioxide and $37^{\circ} \mathrm{C}$. Following this, an appropriate volume of transduction solutions from different experimental groups (LacZ-carrying Bac, LacZ-carrying NP, Bac ${ }_{\text {null }}$-LacZ-carrying NP, LacZcarrying Bac, LacZ-carrying NP, LacZ-carrying Bac-NP ${ }_{\text {null }}$ ), suspended in phosphate buffered saline, was added to each well according to varied multiplicity of infection, and incubated for 4 hours at $25^{\circ} \mathrm{C}$. $\mathrm{Bac}_{\text {null }}$ and $\mathrm{NP}_{\text {null }}$ represent delivery systems carrying DNA with no gene of interest. The wells were replenished with fresh media and grown $37^{\circ} \mathrm{C}$ in carbon dioxide incubator. After 24 hours, the cells were stained with $\mathrm{X}$-gal to detect the transduced LacZ-expressing cells. Results are presented in Figure S2.

\section{Bac-NP has no toxic effects on hASCs: cytotoxicity assay}

For the cytotoxicity assay, $2 \times 10^{4} \mathrm{hASC}$ /well were seeded in triplicate for each sample in 96-well plates. After culturing overnight, the cells were washed twice with phosphate buffered saline. NP, Bac, and Bac-NP particles suspended in culture media $(200 \mu \mathrm{L})$ were added to the corresponding set of wells. Nontransduced cells were used as control. After 24 hours, absorbance of each well were measured at $490 \mathrm{~nm}$ using CellTiter $^{\circledR} 96$ AQueous Non-Radioactive Cell Proliferation Assay (Promega, Fitchburg, WI) in a plate reader. The percentage of viable cells in different experimental 

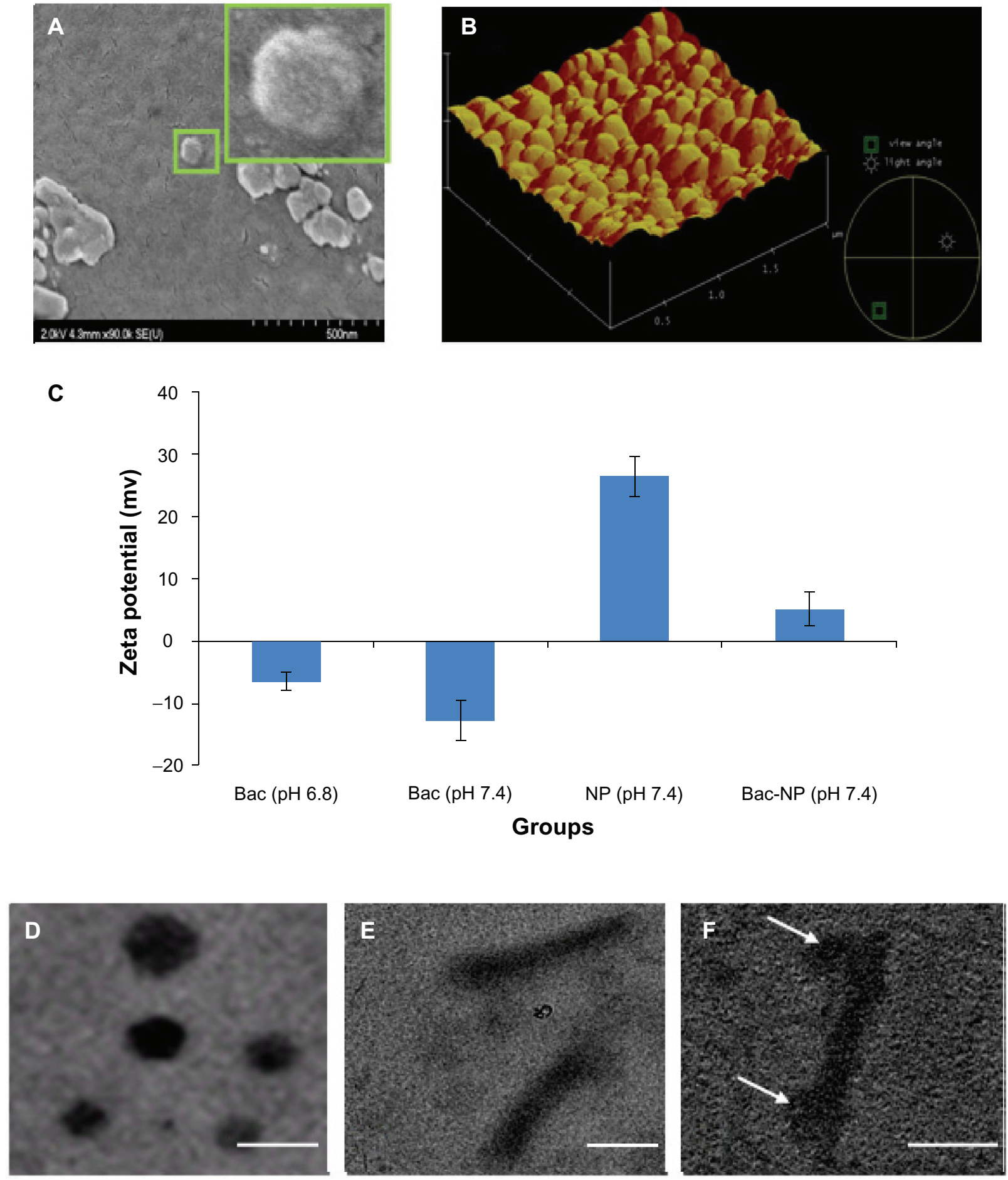

Figure SI Characterization of the Bac-NP hybrid nanocomplex. (A) Scanning electron microscope photograph of transactivating transcriptional activator/deoxyribonucleic acid nanoparticles with subset showing a magnified image. (B) Atomic force microscope photograph of NPs demonstrating their surface topography. (C) Zeta potential of free $\mathrm{Bac}$ at pH 6.8 ( $\mathrm{pH}$ of insect cells media), free Bac at pH 7.4 ( $\mathrm{pH}$ of mammalian cell culture), free NP (phosphate buffered saline: $\mathrm{pH}$ 7.4), and hybrid Bac-NP nanocomplexes at $\mathrm{pH} 7.4$, with N/P ratio of 3. The complexes were prepared from I $\mu \mathrm{g}$ deoxyribonucleic acid (complexed with transactivating transcriptional activator) per $10^{\circ} \mathrm{plaque}$ forming units Bac. Transmission electron microscope images of (D) NP with N/P ratio of 3, (E) Bac, and (F) Bac-NP suspended in phosphate buffered saline.

Notes: Arrows indicate NPs hybridized on Bac surface. Scale bar: $100 \mathrm{~nm}$.

Abbreviations: Bac, baculovirus; Bac-NP, baculovirus-nanoparticle complex; NP, nanoparticle. 


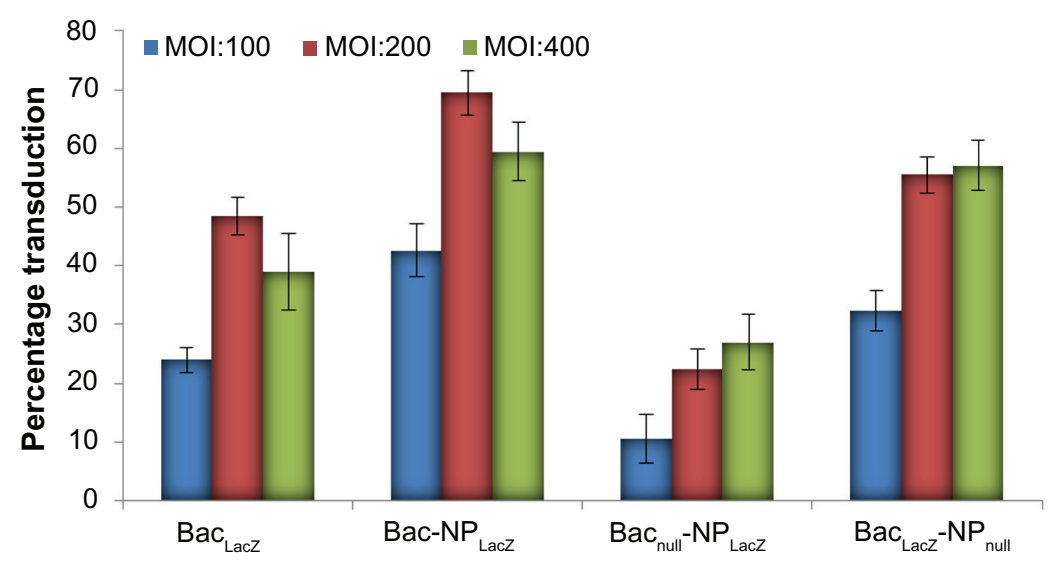

Figure S2 Optimization of transduction condition in human adipose tissue-derived stem cells with Bac-NP cultured overnight. The nanobiohybrid complexes were prepared from I $\mu \mathrm{g}$ deoxyribonucleic acid (complexed with transactivating transcriptional activator) per $10^{9}$ plaque forming units baculovirus. Effect of baculovirus $\mathrm{MOI}$ on baculovirus-nanoparticle complex-mediated cell transduction. Cells were transduced with the prepared baculovirusnanoparticle complex at constant N/P ratio of 3 with $\mathrm{MOI}$ of 100, 200, and 400 and stained with X-gal, 24 hours post-transduction, to determine the percentage cells transduced with LacZ expression. Three independent experiments were performed and data expressed as mean \pm standard deviation. Bac-NP ${ }_{\text {LacZ }}$ with $\mathrm{MOI}_{\mathrm{O}}$ of 200 showed highest transduction as compared to other groups.

Abbreviations: $\mathrm{Bac}_{\mathrm{LacZ}}$, LacZ-carrying baculovirus; $\mathrm{Bac}_{\mathrm{LacZ}}-\mathrm{NP}_{\text {null, }}$ LacZ-carrying baculovirus-nanoparticle (carrying no gene of interest) complex; Bac-NP $\mathrm{LacZ}$, baculovirusLacZ-carrying nanoparticle complex; $\mathrm{Bac}_{\text {null }}-\mathrm{NP}_{\text {LacZ }}$, baculovirus (carrying no gene of interest)-LacZ-carrying nanoparticle complex; MOI, multiplicity of infection.

groups was quantified. All the experiments were performed in triplicate. Results are presented in Figure S3.

\section{Bac-NP transduced hASCs retain their differentiation potential}

Bac-NP-transduced hASCs were then seeded in a 24-well plate at a high confluency of $6 \times 10^{4}$ cells/well. Nontransduced cells were used as control. After 24 hours, the medium was replaced with either adipogenic or osteogenic differentiation medium
(Invitrogen Life Technologies, Carlsbad, CA). Osteogenic differentiation was assessed using Alizarin Red S staining (Invitrogen) after a 21-day period of induction towards this lineage. After a 15-day period, adipogenic differentiation was evaluated by LipidTOX ${ }^{\mathrm{TM}}$ red neutral lipid staining (Invitrogen). Results are presented in Figure S4. The presence of calcium deposits following osteogenic induction, as well as lipid vacuoles following adipogenic induction, is indicative of the transduced hASCs' ability for multilineage differentiation.

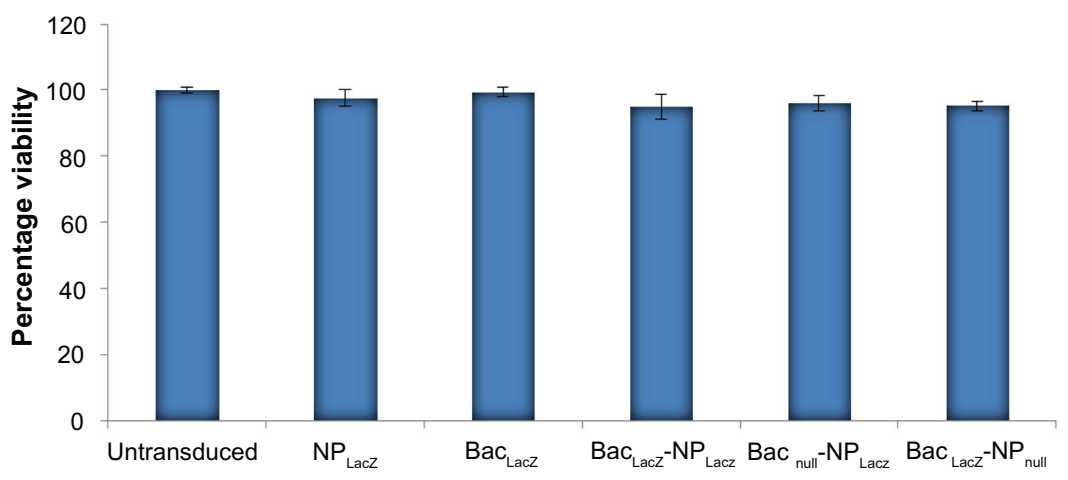

Figure S3 Cytotoxic effects of baculovirus-nanoparticle hybrid complexes on human adipose tissue-derived stem cells. In each well of 96 -well plate, $2 \times 10^{4}$ cells were seeded and cultured overnight. The cells were incubated with nanoparticles (N/P ratio 3) only, baculovirus (multiplicity of infection 200) only, and baculovirus-nanoparticle complex (multiplicity of infection 200 and N/P ratio 3) for 12 hours followed by cell toxicity analysis.

Notes: Three independent experiments were performed and data expressed as mean \pm standard deviation. There were no significant differences in percentage viability between the groups, confirming that the baculovirus-nanoparticle complex did not have any toxic effect on the human adipose tissue-derived stem cells.

Abbreviations: $\mathrm{Bac}_{\mathrm{LacZ}}$, LacZ-carrying baculovirus; $\mathrm{Bac}_{\mathrm{Lac}}-\mathrm{NP}_{\mathrm{LacZ}}$, LacZ-carrying baculovirus-LacZ-carrying nanoparticle complex; Bac ${ }_{\text {Lacz }}-\mathrm{NP}_{\text {null }}$ LacZ-carrying baculovirus-

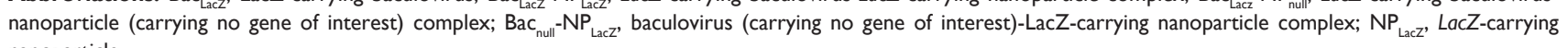
nanoparticle. 

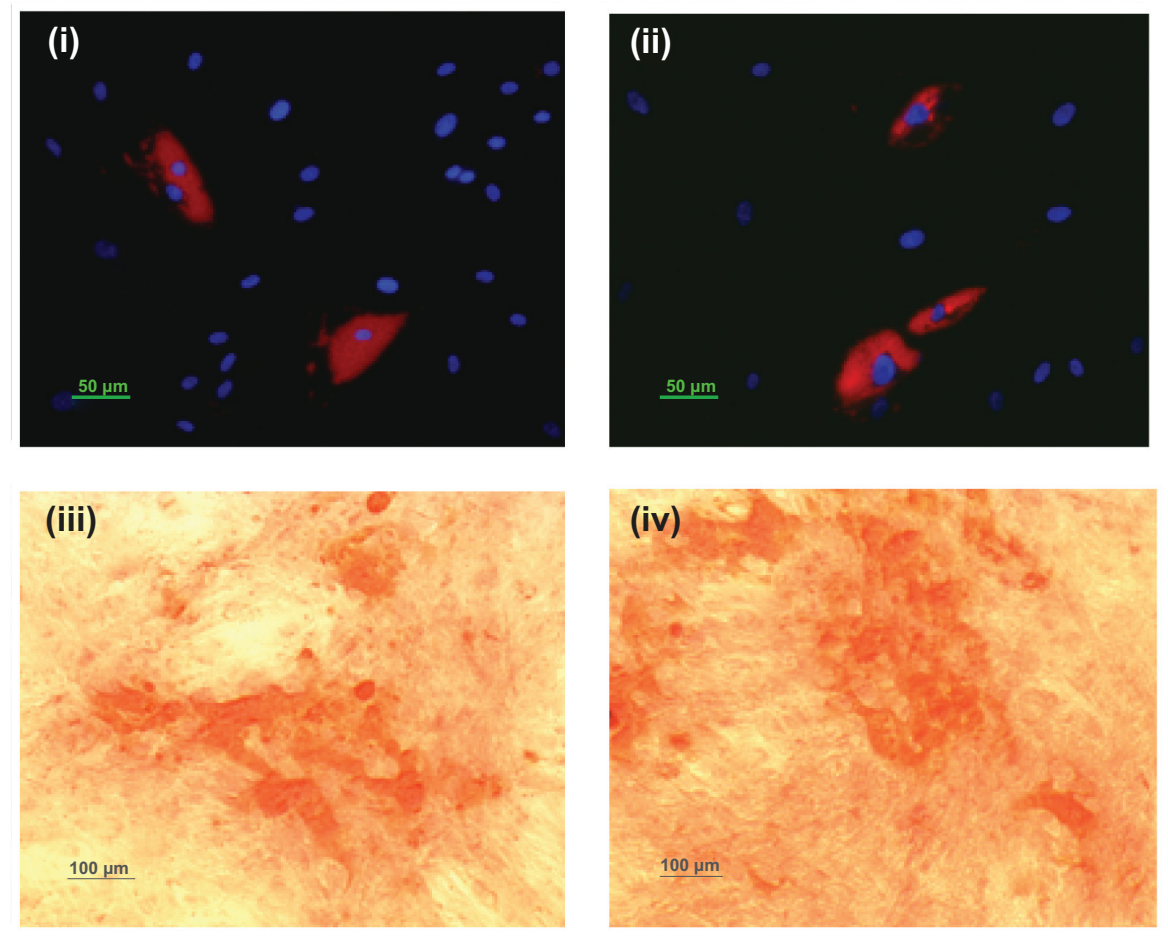

Figure S4 Adipogenic and osteogenic differentiation of baculovirus-nanoparticle complex-transduced human adipose tissue-derived stem cells. Transduced adipose tissue-

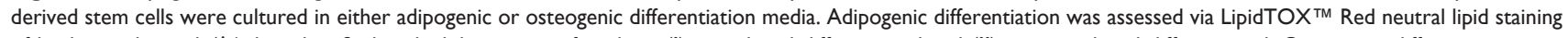
of lipid vacuoles and 4',6-diamidino-2-phenylindole staining of nucleus: (i) transduced differentiated and (ii) nontransduced differentiated. Osteogenic differentiation was determined by Alizarin Red staining of calcium deposits: (i) transduced differentiated and (ii) nontransduced differentiated.

Notes: Arrows show the cell differentiated area. Results confirm that human adipose tissue-derived stem cells retain their multilineage differential potential even after baculovirus-nanoparticle complex transduction.

\section{Publish your work in this journal}

The International Journal of Nanomedicine is an international, peerreviewed journal focusing on the application of nanotechnology in diagnostics, therapeutics, and drug delivery systems throughout the biomedical field. This journal is indexed on PubMed Central, MedLine, CAS, SciSearch ${ }^{\circledR}$, Current Contents ${ }^{\circledR} /$ Clinical Medicine,
Journal Citation Reports/Science Edition, EMBase, Scopus and the Elsevier Bibliographic databases. The manuscript management system is completely online and includes a very quick and fair peer-review system, which is all easy to use. Visit http://www.dovepress.com/ testimonials.php to read real quotes from published authors.

Submit your manuscript here: http://www.dovepress.com/international-journal-of-nanomedicine-journal 